\title{
VARIATIONAL METHOD ON RIEMANN SURFACES USING CONFORMAL PARAMETERIZATION AND ITS APPLICATIONS TO IMAGE PROCESSING*
}

\author{
LOK MING LUI ${ }^{\dagger}$, XIANFENG GU ${ }^{\ddagger}$, TONY F. CHAN ${ }^{\S}$, AND SHING-TUNG YAU
}

\begin{abstract}
Variational method is a useful mathematical tool in various areas of research, especially in image processing. Recently, solving image processing problems on general manifolds using variational techniques has become an important research topic. In this paper, we solve several image processing problems on general Riemann surfaces using variational models defined on surfaces. We propose an explicit method to solve variational problems on general Riemann surfaces, using the conformal parameterization and covariant derivatives defined on the surface. To simplify the computation, the surface is firstly mapped conformally to the two dimensional rectangular domains, by computing the holomorphic 1-form on the surface. It is well known that the Jacobian of a conformal map is simply the scalar multiplication of the conformal factor. Therefore with the conformal parameterization, the covariant derivatives on the parameter domain are similar to the usual Euclidean differential operators, except for the scalar multiplication. As a result, any variational problem on the surface can be formulated to a $2 \mathrm{D}$ problem with a simple formula and efficiently solved by well developed numerical scheme on the $2 \mathrm{D}$ domain. With the proposed method, we solve various image processing problems on surfaces using different variational models, which include image segmentation, surface denoising, surface inpainting, texture extraction and automatic landmark tracking. Experimental results show that our method can effectively solve variational problems and tackle image processing tasks on general Riemann surfaces.
\end{abstract}

Key words. Variational method, conformal parameterization, holomorphic one form, conformal factor, covariant derivative.

AMS subject classifications. 35Q93, 49M25, 58C05, 65K10

1. Introduction. Solving variational problem is an important topic in mathematics. A lot of daily life problems can be solved by formulating them as variational problems that minimize certain kind of energy functionals. This type of problem has a very long history and has found various applications in different research areas such as physics, control theory, statistics as well as image processing. For example, a lot of partial differential equations (PDEs) in physics are derived from the Euler-Lagrange equations of the variational problems. In computer vision research, many problems, such as image denoising and image segmentation, can also be solved by variational approaches [1][2][3][4][5].

Solving variational problems in the usual Euclidean domain has been studied extensively [6]. Recently, researchers have been more and more interested in solving variational problems on general surfaces or manifolds. Applications exist in different areas of research, such as computer vision, computer graphics, image processing on the surface, geometry modeling, medical imaging as well as mathematical physics. In medical imaging research, variational methods are often used for surface registration,

\footnotetext{
* Received April 16, 2009; accepted for publication August 28, 2009.

†Department of Mathematics, Harvard University, Cambridge, MA 02138, USA (malmlui@ math.harvard.edu); and Department of Mathematics, University of California, Los Angeles, CA 90095-1555, USA (malmlui@math.ucla.edu).

${ }^{\ddagger}$ Department of Computer Science, State University of New York at Stony Brook, Stony Brook, NY 11794-4400, USA (gu@cs.sunysb.edu ).

$\S$ Department of Mathematics, University of California, Los Angeles, CA 90095-1555, USA (chan@ math.ucla.edu).

『Department of Mathematics, Harvard University, Cambridge, MA 02138, USA (yau@ math.harvard.edu).
} 
feature extraction, surface parameterization and so on [7][8][9]. Besides, a lot of 2D image processing techniques can be extended to the surface by variational methods on the manifolds, such as image denoising, image inpainting on the surface, brain mapping, etc [10][11][12][13]. Geometry modeling can also be done via variational methods. Examples include surface smoothing, filling missing holes on the surface, etc [14]. In fluid dynamic, researchers are interested in simulating the fluid flow and solidification on the surface, via solving different flow models [15][16].Some other applications include texture synthesis [17][18], vector field visualization [19], weathering [20], interpolation process [21][22] and inverse problem [23]. Therefore, it is of great interest to develop a general and efficient method to solve variational problems on the surface.

In this paper, we describe an explicit method to solve variational problems on general Riemann surfaces, using the conformal parameterization of the surface. In general, variational problem is usually solved by computing its Euler-Lagrange equation, which is essentially a partial differential equation. Therefore, it is important to understand how to do calculus on general manifolds. On Riemann surfaces, differential operations are done through covariant derivatives [24][25][26]. Essentially, they are a set of coordinate invariant operators for taking directional derivatives of the functions or vector fields defined on the surface. Covariant derivatives are defined locally through the local parameterization of the manifold [25]. With arbitrary parameterization, the formulae for the covariant derivatives are generally very complicated. It results in computational difficulties and numerical inaccuracies. Here, we propose to parameterize the surface conformally with the minimum number of coordinates patches. The Riemannian metric of the conformal parameterization is simple, which is just the scalar multiplication of the conformal factor, $\lambda$. The covariant derivatives on the surface can be computed on the $2 \mathrm{D}$ domain with simple formula. The corresponding formula for the covariant derivatives on $R^{2}$ are similar to the usual Euclidean differential operators, except for a scaling factor $\lambda$. Therefore, with the conformal parameterization, the variational problems on general surfaces can be transformed to the $2 \mathrm{D}$ problems with much simpler equations. The problem can then be solved by using some well-known numerical schemes.

The key advantages of this method are as follow:

- Firstly, by mapping the surface to the 2D domain, the problem on the surface are transformed into the $2 \mathrm{D}$ problem. It can then be solved by efficient $2 \mathrm{D}$ numerical methods, instead of solving it on the complicated surface.

- Secondly, the simple Riemannian metric of the conformal parameterization allows us to have a simple formula for the covariant derivatives on the $2 \mathrm{D}$ domain. It makes computation much easier and reduces numerical inaccuracy.

- Thirdly, in our algorithm, covariant derivatives are computed via conformal parameterization without the orthogonal projection of the normal. It is different from some other methods, in which orthogonal projection is needed to ensure the approximated covariant derivatives are tangent to the surface. In our algorithm, the surface is identified with the $2 \mathrm{D}$ parameter domain with a specific Riemannian metric. Every tangent vector of the surface is represented by a $2 \mathrm{D}$ vector in the parameter domain and thus orthogonal projection is unnecessary. It simplifies the problem and avoids possible error arising from the inaccurate approximation of the normal.

- Fourthly, our algorithm allows us to compute the conformal parameterization of the surface with the minimum number of coordinate patches. For most of 
TABLE 1

The list of three common methods that solve variational problems/PDEs on general surfaces.

\begin{tabular}{|l|l|l|}
\hline Method & Principle & Comments \\
\hline \hline $\begin{array}{l}\text { Discretization } \\
\text { on surface tri- } \\
\text { angulation }[10], \\
{[27],[16]}\end{array}$ & $\begin{array}{l}\text { Covariant differential operators on } \\
\text { the surface are approximated by f- } \\
\text { nite element methods on the trian- } \\
\text { gulation grids. }\end{array}$ & $\begin{array}{l}\text { Orthogonal projection is needed in } \\
\text { some cases to ensure the approx- } \\
\text { imated covariant derivatives to be } \\
\text { tangent to the surface. }\end{array}$ \\
\hline $\begin{array}{l}\text { Level set ap- } \\
\text { proach [28], }[29]\end{array}$ & $\begin{array}{l}\text { The surface is represented by the } \\
\text { zero set of a level set function and } \\
\text { the PDE on the surface is extended } \\
\text { to a PDE that is defined on a nar- } \\
\text { row band of the surface. }\end{array}$ & $\begin{array}{l}\text { Equations can be solved by Carte- } \\
\text { sian grid methods on the narrow } \\
\text { band. }\end{array}$ \\
\hline $\begin{array}{l}\text { Surface para- } \\
\text { meterization } \\
{[30],[15],[13]}\end{array}$ & $\begin{array}{l}\text { The surface is parameterized to a } \\
\text { simple domain such as the 2D rec- } \\
\text { tangle. Differential operators on } \\
\text { the surface are expressed within the } \\
\text { coordinates system. }\end{array}$ & $\begin{array}{l}\text { The complexity of the differen- } \\
\text { tial operators' expression depends } \\
\text { mainly on the parameterization, } \\
\text { which may result in more deriva- } \\
\text { tive terms and non-constant coef- } \\
\text { ficients. }\end{array}$ \\
\hline
\end{tabular}

the classical parameterization methods, the surface is segmented into many portions and each portion is mapped to the $2 \mathrm{D}$ parameter domain. In our algorithm, we parameterize the surface with the minimum number of coordinate patches and the parameterization results are consistent along the patch boundaries because of its global parameterization nature. Specifically, the number of coordinate patches is $2 g-2$, where $g$ is the genus of the surface. The parameterization is intrinsic and depends on the holomorphic 1-form, which is in a finite dimensional linear space. Since our segmentation is based on the holomorphic 1-form, the segmentation result is finite and purely determined by holomorphic 1-form selection. Thus we could always select the segmentation that is the most appropriate to solve the PDEs.

- Finally, the conformal metric on the 2D parameter domain is induced by the actual metric of the original surface. As a result, by computing the derivatives on the $2 \mathrm{D}$ domain with respect to the conformal factor, we are computing the actual covariant derivatives on the surface.

2. Related works. Solving variational problems or PDEs on surfaces has been studied extensively. A popular method to solve the PDEs on surfaces is to discretize the problem on the surface triangulation [10][27][16]. In this approach, the covariant differential operators on the surface are approximated by finite element methods on the triangulation grids.

Another common approach is to solve the PDE on the implicit manifold, which is based on the level set method [28][29]. In this approach, the surface is the zero set of level set function defined in $R^{3}$, in which the surface is embedded in. The PDE on the surface is extended to be defined on a narrow band of the surface. Recently, Ratz et al. [31] proposed to solve the PDEs on the surface implicitly by reformulating the problem on a larger domain in one higher dimension and introduce a diffuse interface region of a phase-field variable, which is defined in the whole domain. The surface of interest is now only implicitly given by the $\frac{1}{2}$-level set of this phase-field variable.

Variational problems or PDEs on surfaces can also be solved by parameterizing the surface onto the 2D parameter domain [30][15][13]. Differential operators on the surface are expressed within the coordinates system. The complexity of the differential operators' expression depends mainly on the parameterization, which may result in 

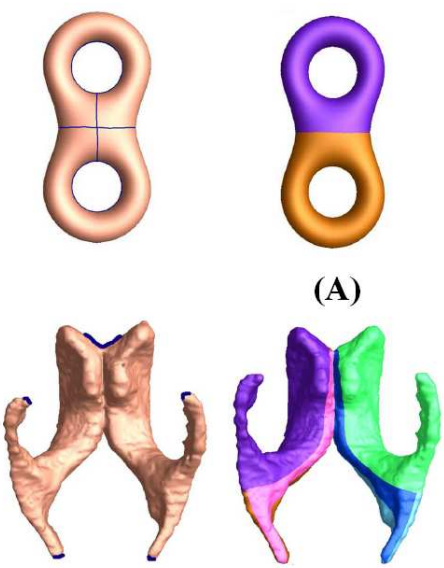

(C)
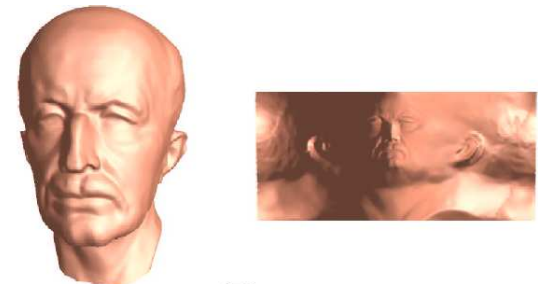

(B)
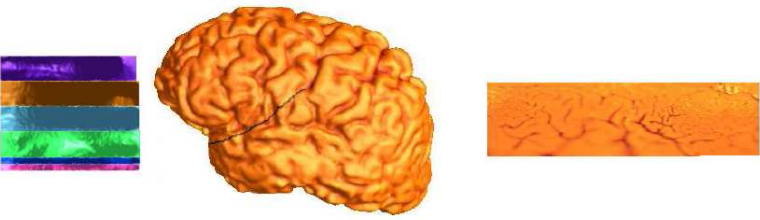

(D)

FIG. 1. Illustration of the conformal parameterizations of different surfaces. The parameterizations are computed by integrating the holomorphic differential one form defined on the surfaces. (A), (B), (C) and (D) show the conformal parameterizations of a 2-torus, a human face, a lateral ventricular surface and a human brain cortical surface [32] respectively.

more derivative terms and non-constant coefficients. To improve this method, our group have recently reported briefly about using conformal mapping to parameterize the surface. The formula of the covariant derivative under conformal parameterization are comparatively simple [13]. To test the method, we have also reported application of the algorithm to feature extraction in the brain mapping research [9][32].

We have summarized the three common parameterization methods in Table 1.

\section{Theoretical background.}

3.1. Conformal parameterization of Riemann surface. All Riemann surfaces are locally Euclidean. Given two Riemann surfaces $M$ and $N$. We can represent them locally as $\phi_{M}\left(x_{1}, x_{2}\right): R^{2} \rightarrow M \subseteq R^{3}$ and $\phi_{N}\left(x_{1}, x_{2}\right): R^{2} \rightarrow N \subseteq R^{3}$ respectively, where $\left(x_{1}, x_{2}\right)$ are their coordinates. The inner product of the tangent vectors at each point of the surface can be represented by its first fundamental form. The first fundamental form on $M$ can be written as $d s_{M}^{2}=\sum_{i, j} g_{i j} d x^{i} d x^{j}$, where $g_{i j}=\frac{\partial \phi_{M}}{\partial x^{i}} \cdot \frac{\partial \phi_{M}}{\partial x^{j}}$ and $i, j=1,2$. Similarly, the first fundamental form on $N$ can be written as $d s_{N}^{2}=\sum_{i, j} \widetilde{g}_{i j} d x^{i} d x^{j}$ where $g_{i j}=\frac{\partial \phi_{N}}{\partial x^{i}} \cdot \frac{\partial \phi_{N}}{\partial x^{j}}$ and $i, j=1,2$. Given a map $f: M \rightarrow N$ between the $M$ and $N$. With the local parameterization, $f$ can be represented locally by its coordinates as $f: R^{2} \rightarrow R^{2}, f\left(x_{1}, x_{2}\right)=\left(f_{1}\left(x_{1}, x_{2}\right), f_{2}\left(x_{1}, x_{2}\right)\right)$. Every tangent vectors $\vec{v}$ on $M$ can be mapped (push forward) by $f$ to a tangent vectors $f_{*}(\vec{v})$ on $N$. The inner product of the vectors $f_{*}\left(\overrightarrow{v_{1}}\right)$ and $\left.f_{*}\left(\overrightarrow{v_{2}}\right)\right)$, where $\overrightarrow{v_{1}}$ and $\overrightarrow{v_{2}}$ are tangent vectors on $M$, is:

$$
\begin{aligned}
f^{*}\left(d s_{N}^{2}\right)\left(v_{1}, v_{2}\right) & :=<f_{*}\left(v_{1}\right), f_{*}\left(v_{2}\right)> \\
& =\sum_{i, j} \widetilde{g}_{i j} f_{*}\left(v_{i}\right) \cdot f_{*}\left(v_{j}\right) \\
& \left.=\sum_{i, j}\left(\sum_{m, n} \widetilde{g}_{m n} \frac{\partial f_{i}}{\partial x^{m}} \frac{\partial f_{j}}{\partial x^{n}}\right) v_{i} v_{j}\right) .
\end{aligned}
$$


Therefore, a new Riemannian metric $f^{*}\left(d s_{N}^{2}\right)$ on $M$ is induced by $f$ and $d s_{N}^{2}$, called the pull back metric. We say that the map $f$ is conformal if

$$
f^{*}\left(d s_{N}^{2}\right)=\lambda\left(x_{1}, x_{2}\right) d s_{M}^{2}
$$

A parameterization $\varphi: R^{2} \rightarrow M$ is a conformal parameterization if $\varphi$ is a conformal map.

Intuitively, a map is conformal if it preserves the inner product of the tangent vectors up to a scaling factor, called the conformal factor $\lambda$. An immediate consequence is that every conformal map preserves angles. Figure 1 shows several examples of conformal parameterization examples. Figure 1(A), 1(B), 1(C) and 1(D) show the conformal parameterizations of a 2 -torus, a human face, a lateral ventricular surface and a brain cortical surface respectively [32].

3.2. Differential operator on general manifolds. The calculus of variation has found various important applications. Differentiation and integration are the basic tools for solving this kind of problem. In order to extend the calculus of variation on the 2D domain to 3D Riemann surface, differential operators on surfaces are needed. On the Riemann surface, differential operators are defined by covariant derivatives. Essentially, they are a coordinate invariant set of operators for taking directional derivatives of the functions or vector fields defined on the surface $S$.

Generally, the covariant derivative $\nabla_{X} F$, where $X$ is a tangent vector on $S, F$ is either a function or a vector field defined on $S$, satisfies the following properties:

(I) $\nabla_{X} F$ is linear in $X$ over $C^{\infty}(S): \nabla_{f X_{1}+g X_{2}} F=f \nabla_{X_{1}} F+g \nabla_{X_{2}} F$.

(II) $\nabla_{X} F$ is linear over $R$ in $F: \nabla_{X}\left(a F_{1}+b F_{2}\right)=a \nabla_{X} F_{1}+b \nabla_{X} F_{2}$.

(III) $\nabla$ satisfies the product rule: $\nabla_{X}(f F)=f \nabla_{X} F+X(f) F$

(Here, we define $X(f)=\left.\frac{d}{d t}\right|_{t=0} f(\alpha(t))$ where $\alpha:(-1,1) \rightarrow S$ is a curve on $S$ such that $\alpha^{\prime}(0)=X$.)

This basically defines the covariant derivatives. $\nabla_{X} F$ is called the covariant derivative of $F$ in the direction $X$.

Let $\varphi: R^{2} \rightarrow S$ be a parameterization of $S$ (not necessarily conformal). Define $g=\left(g_{i j}\right)_{i, j=1,2}$ where $g_{i j}=\varphi_{x_{i}} \cdot \varphi_{x_{j}}$ are the Riemannian metric coefficients. Let $F=F_{1} \varphi_{x_{1}}+F_{2} \varphi_{x_{2}}$ and $X=X_{1} \varphi_{x_{1}}+X_{2} \varphi_{x_{2}}$ be a vector field and a tangent vector on $S$ respectively. Let $f: S \rightarrow R$ be a function defined on $S$. Then:

$$
\begin{aligned}
& \nabla_{X} f=\sum_{i, j, k=1}^{2}\left(g^{i j} \partial_{i} f\right) X_{k} g_{j k} \\
& \nabla_{X} F=\sum_{i, j, k=1}^{2}\left(X F_{k}+X_{i} F_{j} \Gamma_{i j}^{k}\right) \varphi_{x_{k}}
\end{aligned}
$$

where $\Gamma_{i j}^{k}=\Sigma_{l=1}^{2} \frac{1}{2} g^{k l}\left(\partial_{i} g_{j l}+\partial_{j} g_{i l}-\partial_{l} g_{i j}\right)$ and $\left(g^{i j}\right)_{i, j=1,2}$ is the inverse of $g$.

Note that the complexity of the covariant derivative depends solely on the complexity of the Riemannian metric $g$. An arbitrary parameterization might have a very complicated $g$. It is thus important for us to look for a parameterization that gives simple Riemannian metric $g$. It turns out that the conformal mapping is such a map that has simple Rimannian metric.

With covariant derivative, we can define other useful differential operators on $S$, which are analogous to those on $R^{2}$. We are going to list several of them below. 
Firstly, the gradient of the function $f, \nabla_{S} f$, is characterized by the fact that:

$$
X(f)=<\nabla_{S} f, X>_{S}
$$

By simple checking, we get that $\nabla f$ has the following coordinate expression:

$$
\nabla_{S} f=\sum_{i, j=1}^{2} g^{i j} \partial_{i} f \varphi_{x_{j}}
$$

Secondly, we can define divergence on $S$ as follow:

$$
\nabla_{S} \cdot F:=\frac{1}{\sqrt{\operatorname{det}(g)}} \sum_{i=1}^{2} \partial_{i}\left(\sqrt{\operatorname{det}(g)} F_{i}\right)
$$

With the definition of the gradient and the divergence, we can define the Laplacian operator on $S$ as follow:

$$
\begin{aligned}
\triangle_{S} f & :=\nabla_{S} \cdot\left(\nabla_{S} f\right)=\nabla_{S} \cdot\left(\sum_{i, j=1}^{2} g^{i j} \partial_{i} f\right) \\
& =\frac{1}{\sqrt{\operatorname{det}(g)}} \sum_{i=1}^{2} \partial_{i}\left(\sqrt{\operatorname{det}(g)} \sum_{j=1}^{2} g^{j i} \partial_{j} f\right)
\end{aligned}
$$

A more complete development of various differential operators on the surface can be found at [32].

4. Global conformal parameterization of Riemann surface. In order to solve the variational problems on the surface, we firstly parameterize the surface onto the two dimensional parameter domain (see Figure 1). The problem is then solved on the parameter domain, instead of being solved on the original surface. It is desirable to look for a parameterization with simple Riemannian metric to simplify the computation. It turns out conformal parameterization is the best for this purpose.

Surface conformal parameterization has been widely studied. Several researchers [33][34] have proposed different methods to compute the conformal parameterization for topological disk, by introducing the discrete Dirichlet energy, discrete harmonic map, shape-preserving method and so on. Haker et al. [35][36] computed the global conformal map by approximating the Laplace-Beltrami through a linear system. Gu et al. [37] proposed a non-linear variational method to compute the global conformal parameterization of a genus zero surface by minimizing the harmonic map. As for the high genus surface, Gu et al. [38] proposed a method to parameterize the surface to $2 \mathrm{D}$ rectangles by computing its holomorphic 1-form. It is done by approximating the De-Rham cohomology. Recently, Wang et al. [39] systematically analyzed a family of quasi-conformal maps including harmonic maps, conformal maps and least-squares conformal maps with regards to $3 \mathrm{D}$ shape matching. As a result they proposed a shape matching framework by using the least-squares conformal maps. In this paper, we will apply the algorithm by Gu et al. [38][37] to get the conformal parameterization of the surfaces by computing its holomorphic 1-form. We are going to describe briefly the basic idea of this algorithm in this section.

To parameterize a compact surface onto $2 \mathrm{D}$ rectangles, one intuitive technique is to cut it open along some suitable cutting boundaries. For example, a torus of 
genus one can be cut open and mapped to a rectangle along two cutting boundaries. Similarly, a torus of genus two can also be mapped to two rectangles by introducing suitable cut (see Figure 3). If the cut is suitably chosen, the parameterization could be conformal. In the algorithm that we use to parameterize the surface, we search for the suitable cutting boundaries on the surface in order to get a conformal map. This is done by computing the holomorphic one form on the surface. The holomorphic one form $\omega$ is a complex analytic differential form.

To compute the holomorphic 1-form, we start by computing a harmonic 1-form $\omega$ on the surface. Similar to complex analysis, we can compute a harmonic conjugate $* \omega$ of $\omega$, such that $W:=\omega+i * \omega$ is a holomorphic (analytic) 1-form. The basis of harmonic 1-form can be computed from the dual basis of the homology basis. The homology basis is a set of non-constant closed curves (up to homotopic) on the surface. For example, Figure 2 shows the homology basis on different surfaces. (A) shows the homology basis $\left\{e_{1}, e_{2}\right\}$ on a genus one torus. (B) shows the homology basis of a genus two surface, which consists of 4 cutting boundaries. (C) shows the homology basis of a genus four surface, which consists of 8 cutting boundaries. As we cut along the suitable cutting boundaries of the surface, we can map the surface onto the $2 \mathrm{D}$ rectangles.

Given a homology basis $\left\{e_{1}, \ldots, e_{2 g}\right\}$ on the surface, we can compute a set (basis) of the harmonic 1-forms $\left\{\omega_{1}, \ldots, \omega_{2 g}\right\}$ (cohomology) by solving the following system:

$$
\begin{cases}d \omega=\sum_{i=1}^{3} \omega\left(\left[u_{j-1}, u_{j}\right]\right)=0, \forall\left[u_{0}, u_{1}, u_{2}\right] \in M, u_{0}=u_{3} & \text { (closedness); } \\ \triangle \omega=\sum_{[u, v] \in M} \omega([u, v])=0 \forall[u, v] \in M & \text { (harmonicity); } \\ \int_{e_{i}} \omega=\sum_{i=1}^{n_{i}} \omega\left(\left[u_{j-1}^{i}, u_{j}^{i}\right]\right)=\delta_{i j} \forall e_{i}=\sum_{j=1}^{n_{i}}\left[u_{j-1}^{i}, u_{j}^{i}\right], u_{0}^{i}=u_{n_{i}}^{i} & \text { (conjugacy). }\end{cases}
$$

where $\left[u_{0}, u_{1}, u_{2}\right]$ represents a face on $M ;[u, v]$ represents an edge on $M ; k_{u v}=$ $\frac{1}{2}(\cot \alpha+\cot \beta)$ in which $\alpha, \beta$ are the angles against the edge $[u, v]$.

After we get the holomorphic 1-form, we can compute the conformal parameterization $\phi$ by integrating the one form: $\phi(p)=\int_{\gamma} \omega=\int_{\gamma} f\left(z_{\alpha}\right) d z_{\alpha}$, where $\gamma$ is any path joining $p$ to a fixed point $c$ on the surface and $\omega=f\left(z_{\alpha}\right) d z_{\alpha}$.

Double covering techniques are applied to surfaces with boundaries to convert them to closed symmetric surfaces.

Further details about the algorithm can be found in [38], [37].

5. Solving variational problems on Riemann surface with the conformal parameterization. In section III(B), we have described how differential operators are defined on the surface with the given parameterization. With the concept of differentiation and integration on the surface, we can use calculus of variation to solve variational problems on surfaces. In this section, we will describe how covariant derivatives can be easily compued using the conformal parameterization and how it can be applied to solve variational problems.

5.1. Computation of convariant derivatives using conformal parameterization. Given a parameterization of the surface $S$, we can express the surface differential operators within its coordinates. In section III(B), we have discussed how covariant derivatives can be computed with the various formulae defined on $R^{2}$. The formulae consists of the Riemannian metric coefficients $g_{i j}$, which are functions defined on $S$. With an arbitrary parameterization, the Riemannian metric can be complicated. As a result, the equation of the surface differential operators can become substantially complex when written in the coordinate system, involving non-constant coefficients 


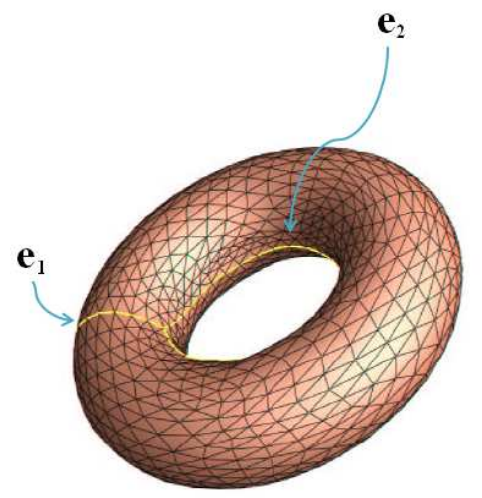

(A)

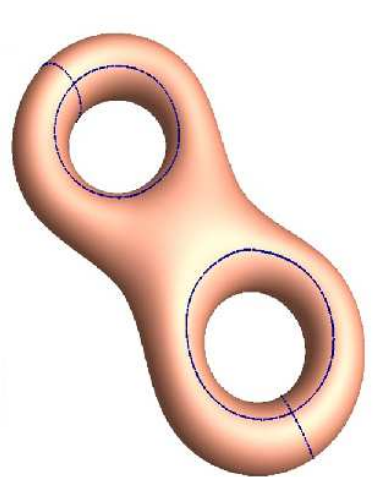

(B)

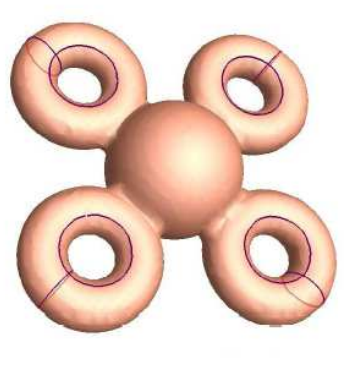

(C)

FIG. 2. Homology basis (cutting boundaries) on different surfaces. (A) shows the homology basis $\left\{e_{1}, e_{2}\right\}$ on a genus one torus. (B) shows the homology basis of a genus two surface, which consists of 4 cutting boundaries. (C) shows the homology basis of a genus four surface, which consists of 8 cutting boundaries.

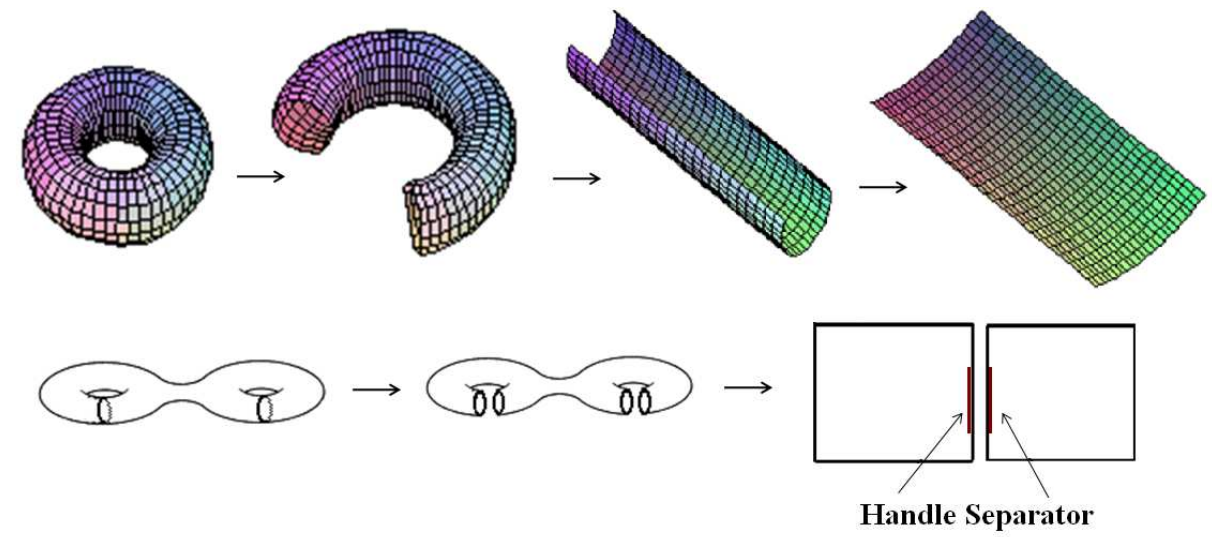

FIG. 3. Illustration of how the conformal parameterization can be computed by introducing suitable cutting boundaries. The top shows how a genus one surface can be mapped to a $2 D$ rectangles by cutting along the suitable cutting boundaries. The bottom shows how a genus two surface (2-torus) can be mapped to two rectangles.

and more derivative terms. Therefore, it is important to look for a parameterization with simple Riemanninan metric.

As described in section III(A), the conformal parameterization has a simple Riemannian metric, namely, $g_{i j}=\left\{\begin{array}{ll}\lambda & \text { if } i=j ; \\ 0 & \text { if } i \neq j .\end{array}\right.$ In other words, the four metric coefficients are reduced to one coefficient metric $\lambda$, called the conformal factor. With this property, surface differential operators can be expressed within the conformal coordinates with simple formulae. The expressions are similar to the usual Euclidean differential operators, except for a scalar multiplication of the conformal factor. The conformal factor at a point $p$ on the surface $S$ can be determined by computing the scaling factor of a small area around $p$ under the parameterization $\phi: R^{2} \rightarrow S$. Mathematically, $\lambda(p)=\frac{\operatorname{Area}\left(B_{\epsilon}(p)\right)}{\operatorname{Area}\left(\phi^{-1}\left(\mathbf{B}_{\epsilon}(\mathbf{p})\right)\right)}$, where $B_{\epsilon}(p)$ is an open ball around $p$ of radius 


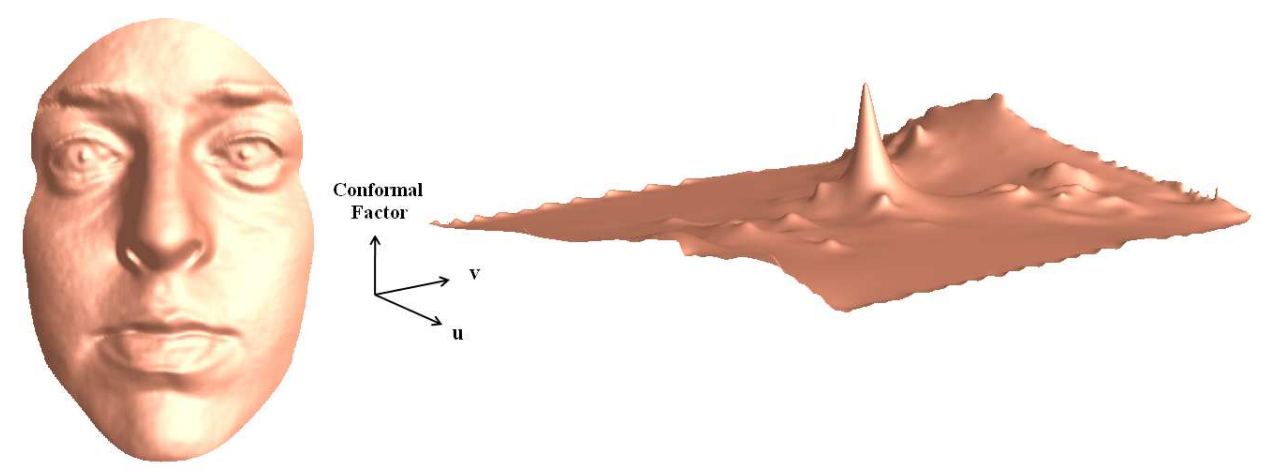

FIG. 4. The plot of the conformal factor $\lambda$ of a human face verses $u$ and $v$ of the parameter domain. The conformal factor is a smooth function which describe the stretching effect under the conformal parameterization. Observe that the approximation of the conformal factor function is reasonably smooth.
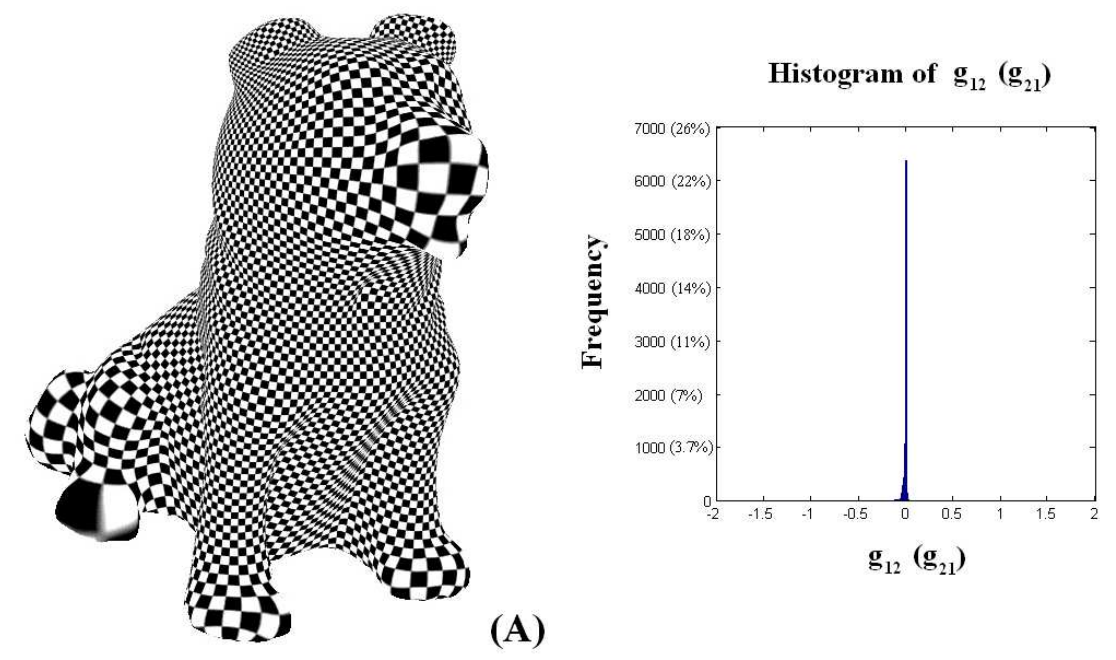

(A)

(B)

FIG. 5. (A) shows the conformal coordinates grid on the dog surface introduced using the conformal parameterization. (B) shows the histogram of $g_{12}=g_{21}$ of a Riemann surface under the conformal parameterization. Observe that $g_{12}=g_{21}$ are very close to zero at most vertex. It means the Riemannian metric is a diagonal matrix, which results in simple expression for the covariant derivatives.

$p$. Figure 4 shows the plot of conformal factor $\lambda$ verses $\mathrm{u}$ and $\mathrm{v}$ of the parameter domain. The conformal factor is a smooth function which describe the stretching effect under the conformal parameterization. Observe that the approximation of the conformal factor function is reasonably smooth. Figure 5(B) shows the histogram of $g_{12}$ of the Riemann surface under the conformal parameterization. Note that by definition, $g_{12}=g_{21}=\phi_{u} \cdot \phi_{v}$, where $\phi(u, v)$ is the conformal parameterization of the surface. Observe that $g_{12}\left(=g_{21}\right)$ are very close to zero at most vertex. It means the Riemannian metric is a diagonal matrix, which results in simple formulae for the covariant derivatives.

We will now express some of the most important surface differential operators under the conformal parameterization $\phi$ of the surface. From section III(B), we have 


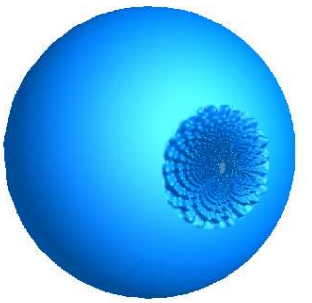

(A)

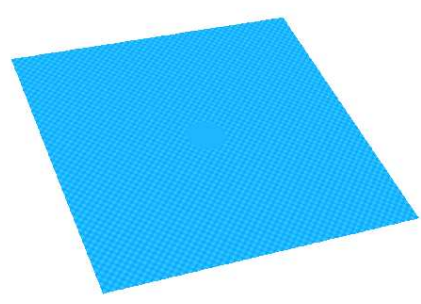

(B)

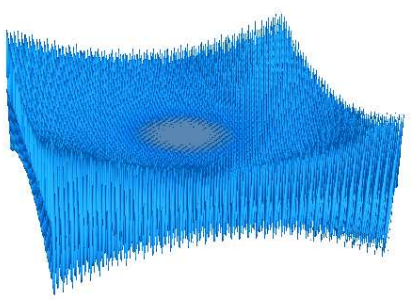

(C)

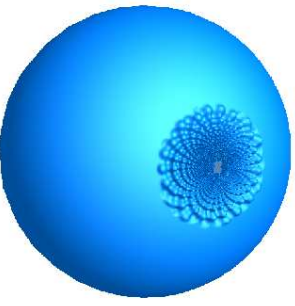

(D)

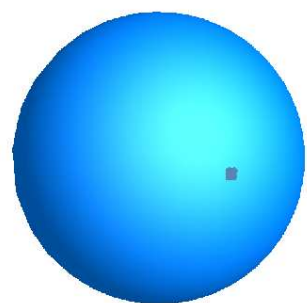

(E)

FIG. 6. This figure demonstrates the importance of including the conformal factor in computing the differential operators on the manifold. (A) shows a unit sphere (minus a hole near the south pole) with noise introduced near the south pole. The surface is parameterized conformally to the 2D parameter domain with large stretching near the south pole. (B) shows the graph of the Eulcidean $T V$ norm of the noise: $T V_{\text {eucl }}(g)=|\nabla g|$. Observe that it does not reflect the noise on the surface due to the strectching effect. (C) shows the manifold version of the TV norm (with conformal factor included): $T V_{\text {manifold }}(g)=\frac{1}{\lambda}|\nabla g|$. It effectively reflects the noise on the surface. (D) shows the denoising result which minimize the Eulcidean TV energy. The noise cannot be removed. (D) shows the denoising result which minimizes the manifold TV norm. The noise is successfully removed.

discussed the expression of $\nabla_{S} f, \triangle_{S} f, \Gamma_{i j}^{k}$ and $\nabla_{X} Y$ under general parameterization of the surface, where $f: S \rightarrow R, X, Y$ are vector fields defined on $S$. Substituting $g_{11}=g_{22}=\lambda ; g_{12}=g_{21}=0$ into the equations (3)(4)(5), we obtained simple formulae for these important surface differential operators within the conformal parameter domain:

$$
\begin{aligned}
& \nabla_{S} f=D_{x} f \overrightarrow{\mathbf{i}}+D_{y} f \overrightarrow{\mathbf{j}} \\
& \operatorname{div}_{S} \vec{X}=\frac{1}{\lambda} \frac{\partial}{\partial x}\left(\lambda X_{1}\right)+\frac{1}{\lambda} \frac{\partial}{\partial y}\left(\lambda X_{2}\right) \\
& \triangle_{S} f=\frac{1}{\sqrt{\lambda}} \frac{\partial^{2}}{\partial x^{2}}(f \circ \phi)+\frac{1}{\sqrt{\lambda}} \frac{\partial^{2}}{\partial y^{2}}(f \circ \phi)
\end{aligned}
$$

where $D_{x} f=\frac{1}{\sqrt{\lambda}} \frac{\partial}{\partial x} f \circ \phi ; D_{y} f=\frac{1}{\sqrt{\lambda}} \frac{\partial}{\partial y} f \circ \phi ; \quad(\overrightarrow{\mathbf{i}}, \overrightarrow{\mathbf{j}})=$ $\left(\frac{\partial}{\partial x} / \sqrt{<\frac{\partial}{\partial x}, \frac{\partial}{\partial x}>_{S}}, \frac{\partial}{\partial y} / \sqrt{<\frac{\partial}{\partial y}, \frac{\partial}{\partial y}>_{S}}\right) ; \quad \frac{\partial}{\partial x}:=\frac{\partial \phi}{\partial x}$ and $\frac{\partial}{\partial y}:=\frac{\partial \phi}{\partial y}$ are the projected tangent vectors of $e_{1}=(1,0)$ and $e_{2}=(0,1)$ onto the surface under the conformal parameterization $\phi$ respectively; $\vec{X}=\left(X_{1}, X_{2}\right)=X_{1} \frac{\partial}{\partial x}+X_{2} \frac{\partial}{\partial y} ; f: S \rightarrow R$ is a smooth function on $S$.

As shown above, the expressions for the surface differential operators are very similar to the usual Euclidean differential operators, except for a scalar multipli- 
cation of the conformal factor $\lambda$. It means the conformal parameterization provides a natural coordinates grid on the surface. Intuitively, the conformal parameterization preserves the inner product up to a scaling factor and so the local geometry is preserved up to a scaling of $\lambda$. As a result, the differentials on $S$ are well-preserved, except for a multiplication of $\sqrt{\lambda}$, to adjust for the length stretching and $\lambda$ for area distortion. With this conformal grid on the surface, we can consider $D_{x} f$ and $D_{y} f$ as the analogous partial derivatives on $S$. For example, $D_{x} f=\lim _{\triangle x \rightarrow 0} \frac{f \circ \phi(x+\triangle x, y)-f \circ \phi(x, y)}{\operatorname{dist}(\phi(x+\Delta x), \phi(x))}=\lim _{\triangle x \rightarrow 0} \frac{f \circ \phi(x+\triangle x, y)-f \circ \phi(x, y)}{\sqrt{\lambda} \triangle x}=\frac{1}{\sqrt{\lambda}} \frac{\partial f \circ \phi}{\partial x}$.

Several important equations that are useful for the calculus of variation on $R^{2}$ are also valid on general surfaces. For example, we have the integration by part formula on surface:

$$
\int_{S}\left(u \triangle_{S} v-v \triangle_{S} u\right) d V=\int_{\partial S}\left(u \nabla_{S} v \cdot \vec{N}-v \nabla_{S} u \cdot \vec{N}\right) d \widetilde{V}
$$

The analogous Green's formula on the surface is:

$$
\int_{S}<\nabla_{S} u, \vec{X}>_{S} d V=-\int_{S} u d i v_{M} \vec{S} d V+\int_{\partial S} u<\vec{X}, \vec{N}>d \widetilde{V}
$$

where $\vec{N}$ is the unit normal vector.

Furthermore, given a smooth function $h: S \rightarrow R$, the length $\mathrm{L}$ of the zero level set $h^{-1}(0)$ of $h$, which is a curve on the surface, can be computed similarly as in $R^{2}$ :

$$
\begin{aligned}
L & =\int_{S} \delta(h) \sqrt{<\nabla_{S} h, \nabla_{S} h>} d A=\int_{S} \sqrt{<\nabla_{S} H(h), \nabla_{S} H(h)>} d A \\
& =\int_{R^{2}} \delta(h \circ \phi) \sqrt{\lambda}\|\nabla h \circ \phi\| d x d y=\int_{R^{2}} \sqrt{\lambda}\|\nabla H(h \circ \phi)\| d x d y
\end{aligned}
$$

where $H$ is the Heaviside function.

The geodesic curvature $G$ of $h^{-1}(0)$ can also be computed as:

$$
G=\operatorname{div}_{S}\left(\frac{\nabla_{S} h}{\left\|\nabla_{S} h\right\|}\right)
$$

similar to the case in $R^{2}$.

5.2. Examples. Since the important equations useful for the calculus of variation on $R^{2}$ can be extended to general surfaces, we can solve the variational problems on the surface easily using the differential operators defined in III(B). In this section, we will demonstrate the theoretical concept by considering two examples.

EXAmple 1 (Harmonic Energy).

Suppose $S$ is a Riemann surface with boundary $\partial S$. Let $\phi: R^{2} \rightarrow S$ be its conformal parameterization. We are interested in looking for a smooth function $f$ : $S \rightarrow R$ that minimizes: $E(f)=\int_{S}\left\|\nabla_{S} f\right\|_{S}^{2} d S$ and $f=0$ on $\partial S$. Consider:

$$
\begin{aligned}
\left.\frac{d}{d t}\right|_{t=0} E(f+t g) & =\int_{S}\left\|\nabla_{S}(f+t g)\right\|_{S}^{2} d S=\int_{S}<\left(\nabla_{S} f+t \nabla_{S} g\right),\left(\nabla_{S} f+t \nabla_{S} g\right)>_{S} d S \\
& =2 \int_{S}<\nabla_{S} f, \nabla_{S} g>_{S} d S=2 \int_{S}\left(\triangle_{S} f\right) g d S .
\end{aligned}
$$


So, the Euler-Lagrange equation is:

$$
\frac{d f^{t}}{d t}=-2 \triangle_{S} f^{t} \text { or } \frac{d f^{t} \circ \phi}{d t}=-\frac{2}{\sqrt{\lambda}} \triangle f^{t} \circ \phi \text { on the parameter domain. }
$$

It is observed that the Euler Lagrange equation of the harmonic energy on the surface is the same as its $2 \mathrm{D}$ version, except that the differential operators in the equation have to be replaced by the manifold operators. The corresponding equation on the parameter domain is similar to its $2 \mathrm{D}$ version on $R^{2}$, except for a scaler multiplication of the conformal factor. When $\lambda=1$, the Riemann surface is flat and so it becomes identical to its $2 \mathrm{D}$ version.

Example 2 (Total Variation).

Suppose now we are interested in looking for a minimizer $f: S \rightarrow R$ of $E(f)=$ $\int_{S}\left\|\nabla_{S} f\right\|_{S} d S$ where $f=0$ on $\partial S$. Consider:

$$
\begin{aligned}
\left.\frac{d}{d t}\right|_{t=0} E(f+t g) & =\int_{S}\left\|\nabla_{S}(f+t g)\right\|_{S} d S=\int_{S} \sqrt{<\left(\nabla_{S} f+t \nabla_{S} g\right),\left(\nabla_{S} f+t \nabla_{S} g\right)>_{S}} d S \\
& =2 \int_{S}<\frac{\nabla_{S} f}{\left\|\nabla_{S} f\right\|_{S}}, \nabla_{S} g>_{S} d S=2 \int_{S} d i v_{S}\left(\frac{\nabla_{S} f}{\left\|\nabla_{S} f\right\|_{S}}\right) g d S .
\end{aligned}
$$

So, the Euler Lagrange equation becomes:

$$
\begin{aligned}
\frac{d f^{t}}{d t} & =-2 \operatorname{div}_{S}\left(\frac{\nabla_{S} f^{t}}{\left\|\nabla_{S} f^{t}\right\|_{S}}\right) \\
\text { or } \frac{d f^{t} \circ \phi}{d t} & =-\frac{2}{\lambda} \operatorname{div}_{S}\left(\frac{\nabla_{S} f^{t} \circ \phi}{\left\|\nabla_{S} f^{t} \circ \phi\right\|_{S}}\right) \text { on the parameter domain. }
\end{aligned}
$$

Again, the Euler Lagrange equation of the Total Variation energy is the same as its $2 \mathrm{D}$ version, except for the replacement of the $2 \mathrm{D}$ differential operators by the manifold differential operators. The corresponding equation on the parameter domain is also similar to its $2 \mathrm{D}$ version on $R^{2}$, except for the scaling of the conformal factor.

In general, the Euler Lagrange equation can be obtained easily from its $2 \mathrm{D}$ version by replacing the $2 \mathrm{D}$ differential operators by the manifold differential operators. The corresponding equation on the $2 \mathrm{D}$ parameter domain is similar to its $2 \mathrm{D}$ version, except for a scaling of the conformal factor.

5.3. The meaning of including the conformal factor. Intuitively, the meaning of scaling the differential operators by the conformal factor $\lambda$ is to adjust the length and area distortion. With the angle preserving property of the conformal parameterization $\phi$, a natural coordinates grid can be introduced on the surface by mapping a regular grid on $R^{2}$ onto the surface (See Figure $5(\mathrm{~A})$ ). However, the grid sizes are different at different points because of the stretching effect of the parameterization. The conformal factor $\lambda$ is defined as the stretching factor of the inner product on the tangent plane of the surface under $\phi$. We can therefore adjust the length and area distortion by $\sqrt{\lambda}$ or $\lambda$. Specifically, the stretching factors of the length and area under $\phi$ are $\sqrt{\lambda}$ and $\lambda$ respectively. In order to have a more accurate approximation of the surface differential operators, we need to scale the usual Euclidean differential operators by $\lambda$. For example, the partial derivative $D_{x} f$ on the surface at the point $\phi(p)$ is: $D_{x} f(\phi(p))=\lim _{\Delta x \rightarrow 0} \frac{\Delta f \circ \phi}{\sqrt{\lambda} \Delta x}=\frac{1}{\sqrt{\lambda}} \frac{\partial f \circ \phi}{\partial x}(p)$. 
Here, the grid size $\Delta x$ is scaled by $\sqrt{\lambda}$ to adjust the length distortion. Similarly, $\Delta_{S} f(\phi(p))=\lim _{\Delta x, \Delta y \rightarrow 0} \frac{\Delta_{x}\left(\Delta_{x} f \circ \phi\right)}{\lambda \Delta x^{2}}+\frac{\Delta_{y}\left(\Delta_{y} f \circ \phi\right)}{\lambda \Delta y^{2}}$.

Also, the surface area differential $d S$ is equal to $\lambda d x d y$, so as to adjust for the area distortion.

To demonstrate the importance of including the conformal factor in the formula of the covariant derivatives, we consider a simple example on the unit sphere. 6(A) shows a unit sphere (minus a hole near the south pole) with noise introduced near the south pole. It is conformally parameterized onto the $2 \mathrm{D}$ parameter domain, with large stretching near the south pole. 6(B) shows the graph of the Eulcidean TV norm of the noise: $T V_{\text {eucl. }}(g)=|\nabla g|$. Observe that it does not reflect the noise on the surface due to the stretching effect near the south pole. $6(\mathrm{C})$ shows the manifold version of the TV norm (with conformal factor included): $T V_{\text {manifold }}(g)=\frac{1}{\lambda}|\nabla g|$. It effectively reflects the noise on the surface. (D) shows the denoising result which minimize the Eulcidean TV energy: $E_{\text {eucl. }}(g)=\int T V_{\text {eucl. }}(g)$. The noise cannot be removed. (D) shows the denoising result which minimizes the manifold TV norm: $E_{\text {manifold }}(g)=\int T V_{\text {eucl. }}(g)$. The noise is successfully removed. This example illustrates the importance of the conformal factor for adjusting the length and area distortion introduced through the conformal parameterization.

\section{Image processing on general Riemann surfaces.}

6.1. Image denoising on the surface. With the advance of the $3 \mathrm{D}$ acquisition systems, images on the surface can be effectively captured and stored as digital data. Nevertheless, noise is inevitably introduced during the transmission process. Therefore, it is of interest to look for an efficient algorithm to denoise the digital image defined on the surface. It has also been widely studied by different research groups [40][41][42][43]. On $R^{2}$, total variation (TV) denoising has been extensively used for image restoration that well-preserves edges [3]. It is then natural to extend the 2D TV denoising model to surfaces. With the conformal parameterization, the TV image denoising model can be easily extended.

On $R^{2}$, the TV model reads:

$$
E_{T V}(u)=\int_{D}\left[|\nabla u|+(u-f)^{2}\right] d x d y
$$

where $f: D \subseteq R^{2} \rightarrow R$ is the noisy gray-level image in $R^{2}$. We will look for a minimizer $u: D \rightarrow R$ of $E_{T V}$ to approximate the original clean image. The EulerLagrange equation of it is:

$$
\frac{d u}{d t}=\operatorname{div}\left(\frac{\nabla u}{|\nabla u|}\right)-2(u-f)
$$

The TV model can also be modified to denoise the color image on $R^{2}$ [4]:

$$
E_{T V \text { color }}(\vec{u})=\int_{D}\left[\sqrt{\sum_{i=1}^{3}\left|\nabla u_{i}\right|^{2}}+|\vec{u}-\vec{f}|^{2}\right] d x d y
$$

where $\vec{f}=\left(f_{1}, f_{2}, f_{3}\right): D \rightarrow R^{2}$ is the noisy color image; $\vec{u}=\left(u_{1}, u_{2}, u_{3}\right): D \rightarrow R^{2}$ is the approximation of the original clean color image. The minimizer of $E_{T V \text { color }}$ can 
be found via its Euler-Lagrange equation:

$$
\frac{d u_{i}}{d t}=\operatorname{div}\left(\frac{\nabla u_{i}}{\sqrt{\sum_{i=1}^{3}\left|\nabla u_{i}\right|^{2}}}\right)-2\left(u_{i}-f_{i}\right) \text { for } i=1,2,3 .
$$

These two models can be extended easily to the surfaces. On the surface, the gray-level TV image denoising model reads:

$$
E_{T V}^{S}(u)=\int_{S}\left[\left\|\nabla_{S} u\right\|_{S}+|u-f|^{2}\right] d S,
$$

where $f: S \rightarrow R$ is a gray-level image defined on the surface. Replacing the Eulcidean differential operators by the modified differential operators in (20), we get the EulerLagrange equation of $E_{T V}^{S}$ :

$$
\begin{gathered}
\frac{d u}{d t}=\operatorname{div}_{S}\left(\frac{\nabla_{S} u}{\left\|\nabla_{S} u\right\|_{S}}\right)-2(u-f) \text { or } \\
\frac{d u \circ \phi}{d t}=\frac{1}{\lambda} \operatorname{div}\left(\sqrt{\lambda} \frac{\nabla u}{|\nabla u|}\right)-2(u \circ \phi-f) \text { on the parameter domain, }
\end{gathered}
$$

where $\phi$ is the conformal parameterization of $S$.

The color TV image denoising model can also be extended to the surface:

$$
E_{T V \text { color }}^{S}=\int_{S}\left[\sqrt{\sum_{i=1}^{3}\left\|\nabla_{S} u_{i}\right\|_{S}^{2}}+\|\vec{u}-\vec{f}\|_{S}^{2}\right] d S
$$

where $\vec{f}=\left(f_{1}, f_{2}, f_{3}\right): S \rightarrow R$ is a color image defined on the surface. By replacing the Euclidean differential operator by the manifold differential operator, we get the Euler-Lagrange equation of $E_{T V \text { color }}^{S}$ :

$$
\begin{array}{r}
\frac{d u_{i}}{d t}=\operatorname{div}_{S}\left(\frac{\nabla_{S} u_{i}}{\sqrt{\sum_{i=1}^{3}|| \nabla_{S} u_{i} \|_{S}^{2}}}\right)-2\left(u_{i}-f_{i}\right) \text { for } i=1,2,3 \text { or } \\
\frac{d u_{i} \circ \phi}{d t}=\frac{1}{\lambda} \operatorname{div}\left(\sqrt{\lambda} \frac{\nabla u_{i} \circ \phi}{\sqrt{\sum_{i=1}^{3}\left|\nabla u_{i} \circ \phi\right|^{2}}}\right)-2 \lambda\left(u_{i} \circ \phi-f_{i} \circ \phi\right) \text { for } i=1,2,3
\end{array}
$$

on the parameter domain.

The two energy functions of the denoising models can be minimized by steepest descent algorithm. Notice that the corresponding Euler-Lagrange equation on the parameter domain is very similar to the $2 \mathrm{D}$ version, except for a scaler multiplication of the conformal factor. These two denoising models can effectively denoise the graylevel and color image defined on the surface, which well-preserves the edges. Figure 7 illustrates the TV color image denoising on a dog surface. The top shows the noisy color image on the dog surface. The denoised color image is shown at the bottom. As shown in the figure, the noise are mostly removed and the reconstructed surface is significantly improved.

6.2. Denoising/Smoothing of Riemann surface. Riemann surfaces are usually obtained by laser scanning or other medical image generation methods such as MRI devices, CT and so on. The surfaces are usually represented as triangular meshes. 


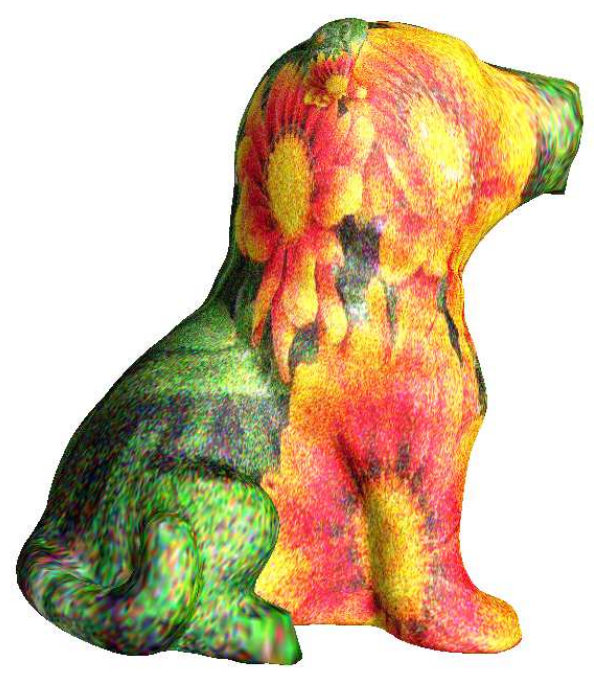

(A)

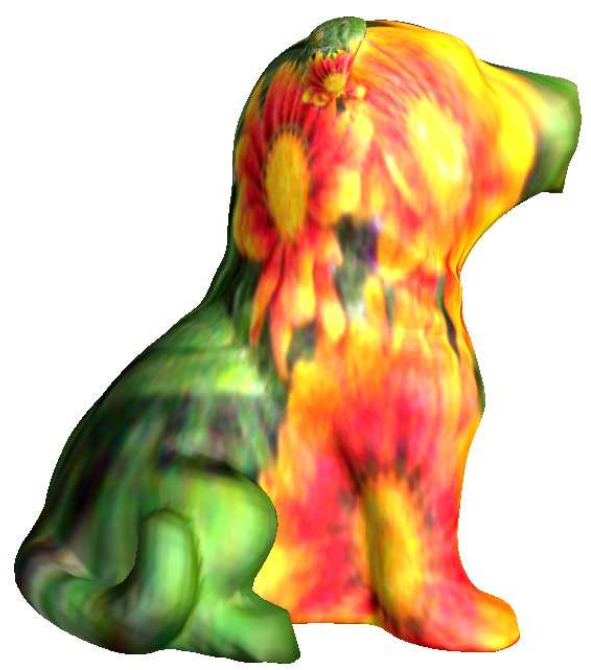

(B)

FIG. 7. Illustration of the TV image denoising on a dog surface. With covariant derivatives, the 2D TV color image denoising model is extended to the 3D Riemann surface. (A) shows the noisy image on the dog surface. (B) shows the denoised image on the surface. As shown in the figure, the noise are mostly removed and the reconstructed surface is significantly improved.

During the construction process, geometric noise is inevitably introduced. Therefore, surface denoising/smoothing, which adjusts the vertices positions so that a smoother surface can be obtained, has become a very important research topic.

Here, we applied the TV denoising technique on the Riemann surface to denoise the noisy surface $S$. Given a conformal parameterization $\phi: R^{2} \rightarrow S$. Let $\vec{\phi}(x, y)=$ $(X(x, y), Y(x, y), Z(x, y))$. The functions $X, Y$ and $Z$ can be regarded as functions defined on the surface $S$. If $S$ is a smooth surface, $X, Y$ and $Z$ are also smooth. By extending the TV denoising technique to the 3D Riemann surface, we can smooth the surface by minimizing the following energy functionals:

$$
E(\vec{\Psi})=\int_{S}|| \nabla_{S} \vec{\Psi} \|_{S} d S+\mu|\vec{\Psi}-\vec{\phi}|^{2}
$$

Or equivalently, we are minimizing the following three energy functionals:

$$
\begin{aligned}
& E(\widetilde{X})=\int_{S}\left\|\nabla_{S} \widetilde{X}\right\|_{S} d S+\mu(\widetilde{X}-X)^{2} \\
& E(\widetilde{Y})=\int_{S}\left\|\nabla_{S} \widetilde{Y}\right\|_{S} d S+\mu(\widetilde{Y}-Y)^{2} \\
& E(\widetilde{Z})=\int_{S}\left\|\nabla_{S} \widetilde{Z}\right\|_{S} d S+\mu(\widetilde{Z}-Z)^{2}
\end{aligned}
$$




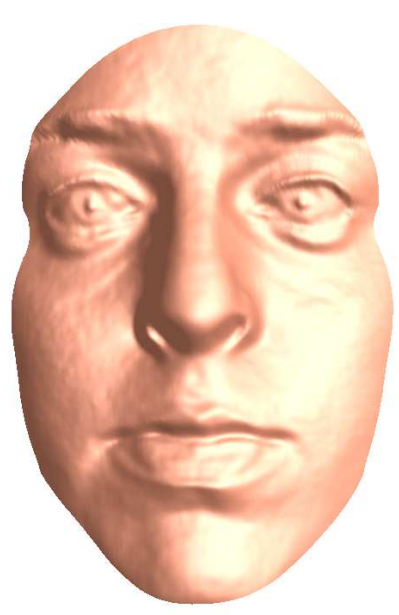

(A)

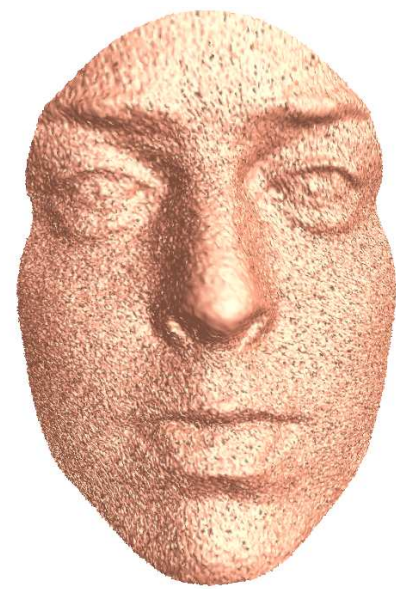

(B)

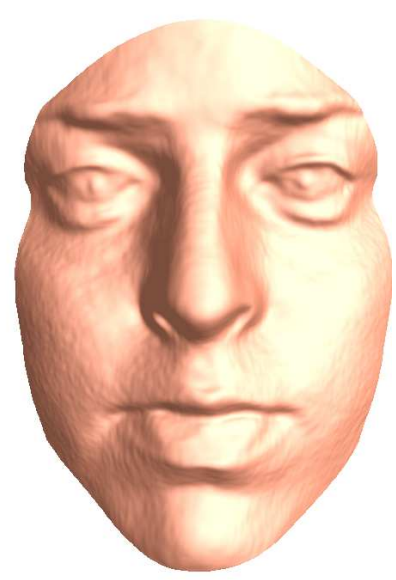

(C)

FIG. 8. Illustration of the TV surface denoising on a human face. With covariant derivatives, the 2D TV image denoising model is extended to 3D Riemann surfaces. (A) shows the original surface of a human face. In (B), the random gaussian noise is added to the face. (C) shows the denoised/smoothed surface. As shown in $(C)$, the reconstructed surface approximates the original surface very well, except for a little bit smoothing.

The Euler Lagrange equations of them are:

$$
\begin{aligned}
& \frac{\partial \widetilde{X}}{\partial t}=\nabla_{S} \cdot\left(\frac{\nabla_{S} \widetilde{X}}{\left\|\nabla_{S} \widetilde{X}\right\|_{S}}\right)+2 \mu(\tilde{X}-X) \\
& \frac{\partial \widetilde{Y}}{\partial t}=\nabla_{S} \cdot\left(\frac{\nabla_{S} \widetilde{Y}}{\left\|\nabla_{S} \widetilde{Y}\right\|_{S}}\right)+2 \mu(\tilde{Y}-Y) \\
& \frac{\partial \widetilde{Z}}{\partial t}=\nabla_{S} \cdot\left(\frac{\nabla_{S} \widetilde{Z}}{\left\|\nabla_{S} \widetilde{Z}\right\|_{S}}\right)+2 \mu(\widetilde{Z}-Z)
\end{aligned}
$$

With the conformal parameterization $\phi$, we can solve these partial differential equations on the $2 \mathrm{D}$ domain with the following three equations:

$$
\begin{aligned}
& \frac{\partial \tilde{X} \circ \phi}{\partial t}=\frac{1}{\lambda} \nabla \cdot\left(\sqrt{\lambda} \frac{\nabla \widetilde{X} \circ \phi}{\|\nabla \widetilde{X} \circ \phi+\epsilon\|}\right)+2 \mu(\widetilde{X} \circ \phi-X \circ \phi) ; \\
& \frac{\partial \widetilde{Y} \circ \phi}{\partial t}=\frac{1}{\lambda} \nabla \cdot\left(\sqrt{\lambda} \frac{\nabla \widetilde{Y} \circ \phi}{\|\nabla \widetilde{Y} \circ \phi+\epsilon\|}\right)+2 \mu(\widetilde{Y} \circ \phi-Y \circ \phi) ; \\
& \frac{\partial \widetilde{Z} \circ \phi}{\partial t}=\frac{1}{\lambda} \nabla \cdot\left(\sqrt{\lambda} \frac{\nabla \widetilde{Z} \circ \phi}{\|\nabla \widetilde{Z} \circ \phi+\epsilon\|}\right)+2 \mu(\widetilde{Z} \circ \phi-Z \circ \phi)
\end{aligned}
$$

where $\lambda$ is the conformal factor of $\phi . \epsilon$ is a small regularization constant to handle with the case when $\|\nabla X \circ \phi\|=0,\|\nabla Y \circ \phi\|=0$ or $\|\nabla Z \circ \phi\|=0$. In practice, we usually take $\epsilon=0.01$. We note that the Euler Lagrange equations are very similar to well-known 2D TV denoising equation, except for the scalar multiplication of the conformal factor $\lambda$. Therefore, we can solve the problem by simple modification of the existing 2D TV denoising solver. 
Figure 8 illustrates the idea of surface denoising/smoothing on a human face with our method. Figure 8(A) shows the original human face surface. In Figure 8(B), the random gaussian noise is added to the surface of the human face. In Figure $8(\mathrm{C})$, we applied the method we described to denoise the surface. The parameter chosen are: $\mu=5, \epsilon=0.01$ and the number of iterations is 50 . Note that the denoised surface approximates the original surface well, except for a little bit smoothing.

The intuitive meaning of including the conformal factor $\lambda$ into the TV model is that it fixes the distortion caused by the stretching. Basically, the TV model denoises the data by smoothing out the high frequency (rapid jump) region. Due the the stretching of the conformal parameterization, the low frequency regions might become the high frequency region in the parameter domain, whereas the high frequency regions become the low frequency region in the parameter domain. As a result, the low frequency regions will be smoothed out and a wrong denoising result will be obtained.

6.3. Texture extraction on the surface. The extraction of features on surfaces has been studied widely [44] and has found various applications in different areas of research. For example, texture extraction is an important process in texture synthesis to transfer textures from one surface to another. In human brain mapping, the feature extraction technique is used to detect important anatomical features on the brain surface to study brain diseases. In this section, we will describe an effective variational method for feature extraction, using the Chan-Vese segmentation model on the surface.

The algorithm consists of two steps. Firstly, we compute the feature intensity on the surface, which encodes the feature information. The feature intensity $I_{f}: S \rightarrow R$ is a function on $S$ defined as:

$$
I_{f}(\phi(p))=\left|\phi(p)-\phi_{\text {smooth }}(p)\right|^{2},
$$

where $\phi_{\text {smooth }}: R^{2} \rightarrow S$ represents the smoothed surface of $S$, using the TV surface smoothing algorithm introduced in VI(B). Specifically, $\phi_{\text {smooth }}$ is obtained iteratively using the gradient descent algorithm to minimize the energy functional in the TV surface smoothing model (See equation ). The feature intensity $I_{f}$ effectively represents the feature information on the surface.

After computing the feature intensity $I_{f}$, the second step is to extract the feature with the Chan-Vese segmentation model on the surface, using $I_{f}$ as the intensity. We proceed to look for $\psi: S \rightarrow R$ that minimizes the following energy functional:

$F\left(c_{1}, c_{2}, \psi\right)=\int_{S}\left(I_{f}-c_{1}\right)^{2} H(\psi) d S+\int_{S}\left(I_{f}-c_{2}\right)^{2}(1-H(\psi)) d S+\nu \int_{S}\left|\nabla_{S} H(\psi)\right| d S$.

The Euler Lagrange equation is:

$$
\begin{array}{r}
\frac{\partial \psi}{\partial t}=\lambda \delta(\psi)\left[\nu \operatorname{div}_{S}\left(\frac{\nabla_{S} \psi}{\left\|\nabla_{S} \psi\right\|_{S}}\right)-\left(I_{f}-c_{1}\right)^{2}-\left(I_{f}-c_{2}\right)^{2}\right] \text { or } \\
\frac{\partial \psi \circ \phi}{\partial t}=\lambda \delta(\psi \circ \phi)\left[\nu \frac{1}{\lambda} \operatorname{div}\left(\sqrt{\lambda} \frac{\nabla \psi \circ \phi}{|\nabla \psi \circ \phi|}\right)-\left(I_{f} \circ \phi-c_{1}\right)^{2}-\left(I_{f} \circ \phi-c_{2}\right)^{2}\right],
\end{array}
$$

on the parameter domain, where:

$$
\begin{array}{r}
c_{1}=\frac{\int_{D} I_{f} \circ \phi(x, y) H(\psi \circ \phi(x, y)) \lambda(x, y) d x d y}{\int_{D} H(\psi \circ \phi(x, y)) \lambda(x, y) d x d y} \\
c_{1}=\frac{\int_{D} I_{f} \circ \phi(x, y)(1-H(\psi \circ \phi(x, y))) \lambda(x, y) d x d y}{\int_{D}(1-H(\psi \circ \phi(x, y))) \lambda(x, y) d x d y} .
\end{array}
$$



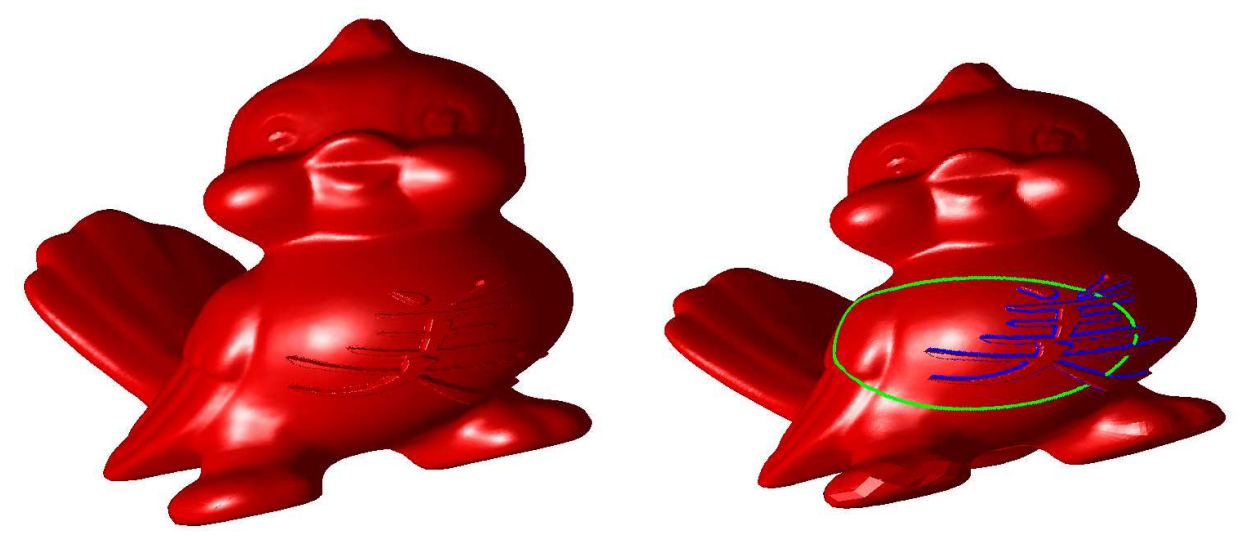

FIG. 9. Illustration of the extraction of texture on the surface. With covariant derivatives, the $2 D$ Chan Vese $(C V)$ segmentation model is extended to $3 D$ Riemann surfaces. The left shows the a bird surface with some texture (chinese character) on it. On the right, we applied the CV segmentation model to extract the texture. The intensity is defined as the distance between the original surface and the smoothed out surface. As shown in the figure, the initial contour (green) evolves to the final contour (blue) that encloses the texture in few iterations.

The zero level set of the minimizer $\psi: S \rightarrow R$ encloses the boundary of the feature on the surface effectively. To illustrate the idea, we apply the algorithm to extract the Chinese character on the cylinder. Figure 9 (left) shows the a surface with some texture (chinese character) on it. In Figure 9(right), we applied our proposed algorithm to extract the feature. The intensity is defined as the distance between the original surface and the smoothed out surface. As shown in the figure, the initial contour (green) evolves to the final contour (blue) that encloses the texture in few iterations.

6.4. Automatic landmark tracking on brain cortical surfaces. One important problem in human brain mapping research is to locate the important anatomical features. Anatomical features on the cortical surface are usually represented by landmark curves, called sulci/gyri curves. These landmark curves are important information for neuroscientists to study brain disease and to match different cortical surfaces. Manual labelling of these landmark curves is time-consuming, especially when large sets of data have to be analyzed. Therefore, an automatic or semi-automatic way to detect these feature curves is necessary. We can automatically detect the sulcal landmarks by solving variational problems on the brain surface [45].

6.4.1. Extraction of sulcal region by Chan-Vese segmentation. In order to speed up the landmark tracking algorithm, we begin with extracting the sulcal regions on the brain surface. This is done by extracting the high mean curvature regions on the cortical surface using the Chan-Vese (CV) segmentation method as described in section $\mathrm{VI}(\mathrm{C})$. The variational model is the same as Equation 25, except that the intensity here is chosen to be the mean curvature defined on the surface. After the sulcal region is extracted, we pick an arbitrary curve lying within the sulcal region as an initial guess of the sulcal landmark.

Figure 10 shows how we can effectively locate the initial landmark guess areas on the cortical surface using the Chan-Vese segmentation. We consider the intensity term 


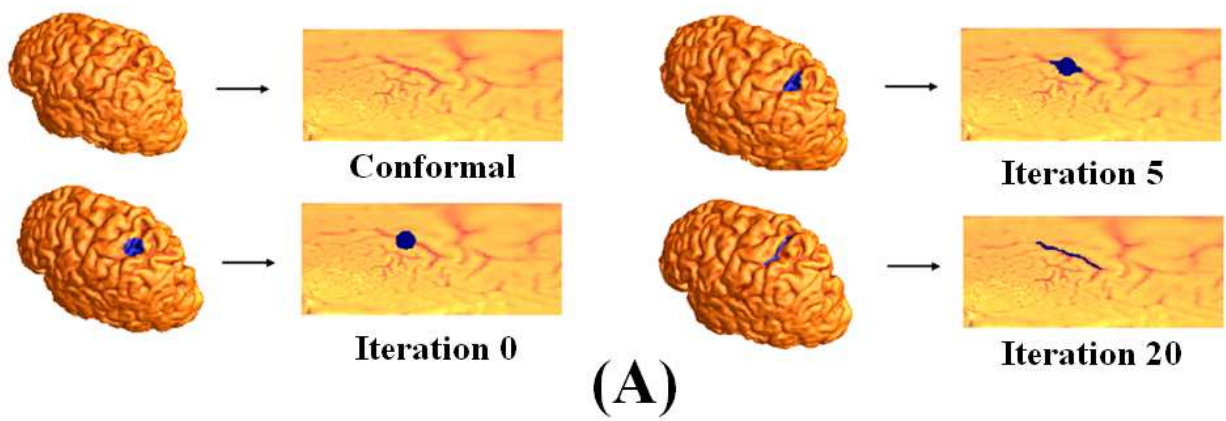

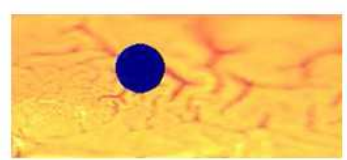

Iteration 0

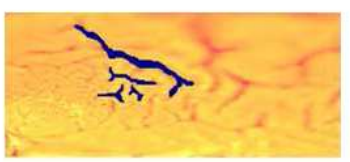

Final iteration

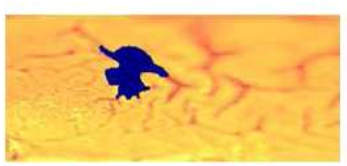

Iteration $\mathbf{5 0}$

\section{Conformal}

Map

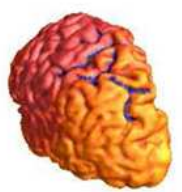

(B)

FIG. 10. Sulcal region extraction on the cortical surface by Chan-Vese segmentation. We consider the intensity term as being defined by the mean curvature. Sulcal locations can then be circumscribed by first extracting out the high curvature regions. (A) shows the result of extraction using a circular initial contour. (B) shows the result of extration using a larger initial circular contour. More sulcal regions can be extracted

as being defined by the mean curvature. Sulcal locations can then be circumscribed by first extracting out the high curvature regions. (A) shows the result of extraction using a circular initial contour. Notice that the contour evolved to the deep sulcal region. (B) shows the result of extration using a larger initial circular contour. More sulcal regions can be extracted.

6.4.2. Variational method for landmark tracking. After the sulcal region is extracted, we can get an initial guess of the sulcal landmark by choosing an arbitrary curve joining the two feature points (umbilic points). This initial guess may not be the most accurate approximation of the sulcus and may not lie on the deepest region. We can iteratively improve the curve such that it moves to the deepest valley of the sulcal region. This is done by a variational approach to get a minimizing curve that follows the principal curvature as much as possible. We have found that the principal directions can effectively be used to trace the sulci and gyri.

The principal direction field $\vec{V}(t)$ with smaller eigenvalues on the cortical surface $C$ can be computed as described in Section. With $\vec{V}(t)$, we propose a variational method to trace the sulcal landmark curve iteratively, after fixing two anchor points $(a \& b)$ on the sulci. Let $\phi: D \rightarrow C$ be the conformal parametrization of $\mathrm{C},\langle\cdot, \cdot\rangle$ to be its Riemannian metric and $\lambda$ to be its conformal factor. We propose to locate a curve $\vec{c}:[0,1] \rightarrow C$ with endpoints $a$ and $b$, that minimizes the following energy 
functional:

$$
E_{\text {principal }}(\vec{c})=\int_{0}^{1}\left|\frac{\overrightarrow{c^{\prime}}}{\sqrt{\left\langle\vec{c}^{\prime}, \vec{c}^{\prime}\right\rangle_{M}}}-\vec{V} \circ \vec{c}\right|_{M}^{2} d t=\int_{0}^{1}\left|\frac{\vec{\gamma}^{\prime}}{\left|\vec{\gamma}^{\prime}\right|}-\vec{G}(\vec{\gamma})\right|^{2} d t
$$

where $\vec{\gamma}=\vec{c} \circ \phi^{-1}:[0,1] \rightarrow D$ is the corresponding iteratively defined curve on the parameter domain; $\vec{G}(\vec{\gamma})=\sqrt{\lambda(\vec{\gamma})} \vec{V}(\vec{\gamma}) ;|\cdot|_{M}^{2}=<\cdot, \cdot>_{M}$ and $|\cdot|$ is the (usual) length defined on D. By minimizing the energy $E$, we minimize the difference between the tangent vector field along the curve and the principal direction field $\vec{V}$. The resulting minimizing curve is the curve that is closest to the curve traced along the principal direction (See Figure 11). Let:

$$
\begin{gathered}
\vec{G}=\left(G_{1}, G_{2}, G_{3}\right) ; \vec{K}=\left(K_{1}, K_{2}, K_{3}\right)=\frac{\vec{\gamma}^{\prime}}{\left|\vec{\gamma}^{\prime}\right|}-\vec{G}(\vec{\gamma}) \\
\vec{L}_{1}=\frac{(1,0,0)}{\left|\vec{\gamma}^{\prime}\right|}-\frac{\gamma_{1}^{\prime} \vec{\gamma}}{\left|\vec{\gamma}^{\prime}\right|^{3}} ; \vec{L}_{2}=\frac{(0,1,0)}{\left|\vec{\gamma}^{\prime}\right|}-\frac{\gamma_{2}^{\prime} \vec{\gamma}}{\left|\vec{\gamma}^{\prime}\right|^{3}} ; \vec{L}_{3}=\frac{(0,0,1)}{\left|\vec{\gamma}^{\prime}\right|}-\frac{\gamma_{3}^{\prime} \vec{\gamma}}{\left|\vec{\gamma}^{\prime}\right|^{3}} .
\end{gathered}
$$

Based on the Euler-Lagrange equation, we can locate the landmark curve iteratively using the steepest descent algorithm:

$$
\frac{d \vec{\gamma}}{d t}=\Sigma_{i=1}^{3}\left[K_{i} \vec{L}_{i}\right]^{\prime}+K_{i} \nabla G_{i}
$$

Our variational method to locate landmark curves is illustrated in Figure 11. With the initial guess given by the Chan-Vese model (we choose the two extreme points in the located area as the anchor points), we trace the landmark curves iteratively based on the principal direction field. In Figure 11 (left), we trace the landmark curves on the parameter domain along the edges whose directions are closest to the principal direction field. The corresponding landmark curves on the cortical surface is shown. Figure 11 (left) shows how the curve evolves to a deeper sulcal region with our iterative scheme. In Figure 11 (right), ten sulcal landmarks are located using our algorithm.

6.5. Inpainting surface holes. 3D surface model are usually obtained from range scanners. Very often, surfaces obtained from range scanner have holes and so resulting in incomplete surface meshes. This may be due to low reflectance, occlusion, scanner placement, inadequate coverage of the object and so on. Recently, reseachers have been interested in inpainting surface holes to reconstruct the incomplete surface and it has become an important research topic [14][46]. In this section, we present an algorithm to solve this problem which involves solving PDEs on the surface.

Inpainting can be regarded as a process of interpolating data on the occluded region from the known data on its neighborhood. Our algorithm is an extension of $2 \mathrm{D}$ image inpainting. To inpaint an occlude 2D digital image, we can fill in the missing region by solving the Perona-Malik diffusion model that reads:

$$
\begin{cases}\frac{\partial u}{\partial t}=\operatorname{div}(g(|\nabla u|) \nabla u) & \text { on } D \\ u^{0}=v & \text { on } D^{c}\end{cases}
$$

where $D$ is the occluded region; $v: D^{c} \rightarrow R$ is the original image with occlusion; $u$ is the approximated (inpainted) image; $g: R \rightarrow R$ is an increasing function such that 

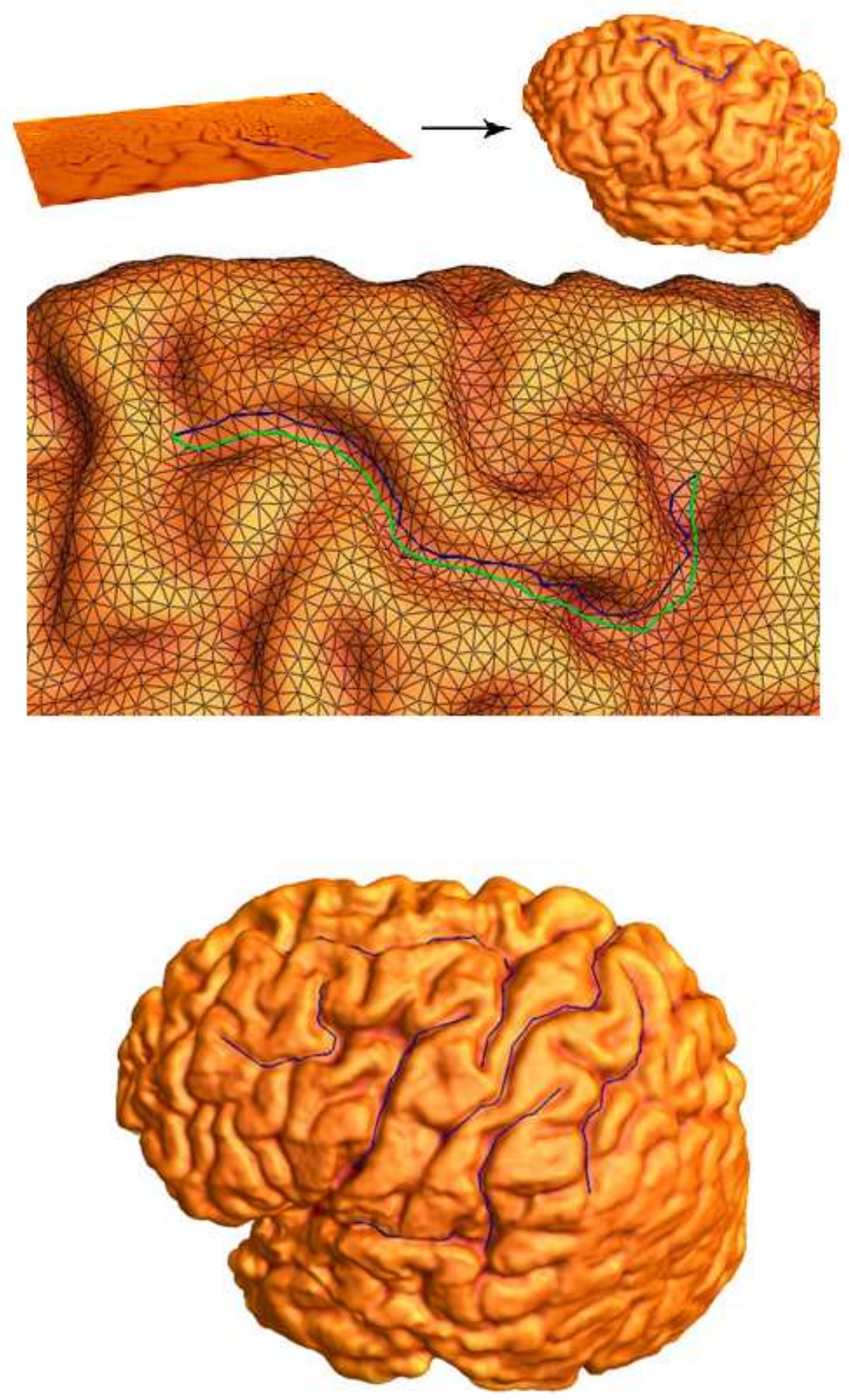

FIG. 11. Automatic landmark tracking using a variational approach. Top : With the global conformal parameterization of the entire cortical surface, we trace the landmark curves on the parameter domain along the edges whose directions are closest to the principal direction field. It gives a good initial guess of the landmark curve (blue curve). The landmark curve is then evolved to a deeper region (green curve) using our variational approach. Bottom: Ten sulcal landmarks are automatically traced using our algorithm. 


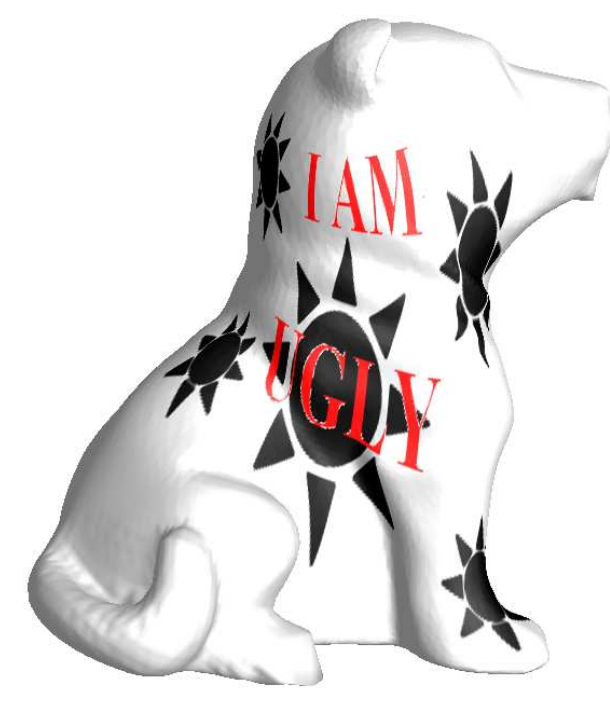

(A)

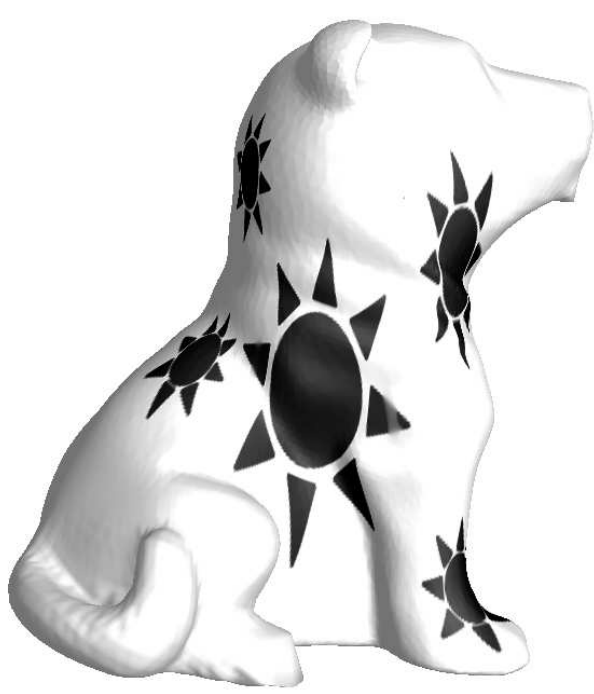

(B)

FIG. 12. "I am not ugly! Please remove the bad words on my body." Illustration of image inpainting on the dog surface. We extend the $2 D$ image inpainting model to $3 D$ surface to remove unwanted words on the dog surface. (A) shows a dog surface with some unwanted words on it. We applied the image inpainting model to inpaint the image. (B) shows the inpainted result. As shown in the figure, the words are successfully removed.

$g(0)=0$ and $g(\infty)=\infty$. Note that if we replace $g$ by $\frac{1}{\nabla u}$, we get the familiar TV smoothing model. The TV smoothing model is well-known to be preserving edges. The major drawback of it is that it does not restore well a single object when its disconnected remaining parts are separated far apart by the inpainting domain. In order to solve this problem, we add the function $g$ into the diffusion model which enhances the diffusion across the boundary of the inpainting domain. Sometimes, it may be more beneficial to let $g$ depend on the curvature $\kappa=\operatorname{div}\left(\frac{\nabla u}{|\nabla u|}\right)$ and so the model depends on the geometry of the image. Here in our application to inpaint surface holes, we have found that letting $g$ depend on the isophote $|\nabla u|$ is already good enough to get a reasonably good result.

The 2D inpainting model can be easily extended to surfaces by using our algorithm. Figure 12 shows the image inpainiting result on the dog surface. To inpaint surface holes, we apply the image inpainting model on the surface $S$. Again, we can regard $X: S \rightarrow R, Y: S \rightarrow R$ and $Z: S \rightarrow R$ as three smooth functions defined on $S$. Given the conformal parameterization $\phi: R^{2} \rightarrow S$ of $S$, we can have the following surface holes inpainting model:

$$
\begin{cases}\frac{\partial X^{t}}{\partial t}=\operatorname{div}_{S}\left(g\left(\|\nabla X\|_{S}\right) \nabla_{S} X\right) & \text { on } D_{S} \\ \frac{\partial Y^{t}}{\partial t}=\operatorname{div}_{S}\left(g\left(\|\nabla Y\|_{S}\right) \nabla_{S} Y\right) & \text { on } D_{S} \\ \frac{\partial Z^{t}}{\partial t}=\operatorname{div}_{S}\left(g\left(\|\nabla Z\|_{S}\right) \nabla_{S} Z\right) & \text { on } D_{S} \\ X^{0}=X_{v} & \text { on } D_{S}^{c} \\ Y^{0}=Y_{v} & \text { on } D_{S}^{c} \\ Z^{0}=Z_{v} & \text { on } D_{S}^{c}\end{cases}
$$




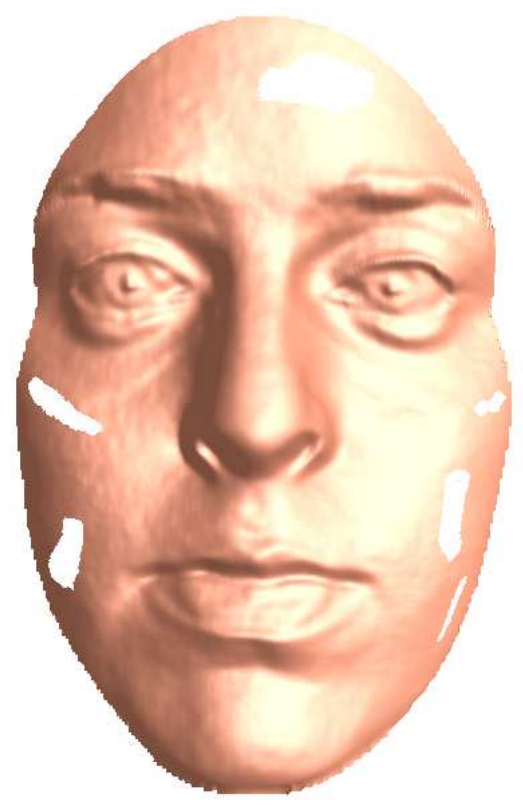

(A)

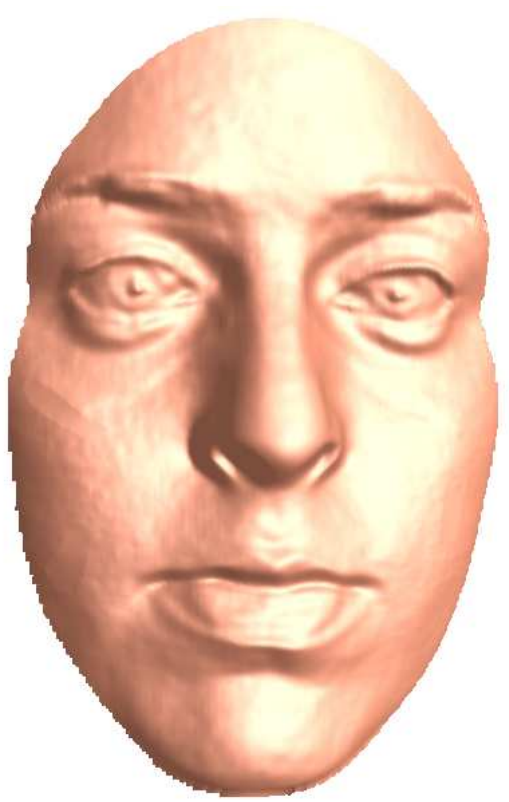

(B)

FIG. 13. Illustration of the algorithm for inpainting surface holes. We extend the 2D image inpainting model to 3D surface to fill in surface holes. (A) shows a human face with several holes on it. We applied the surface holes inpainting model to inpaint the occlusion region on the surface. (B) shows the inpainted result. As shown in the figure, the occlusion can be filled reasonably well, which results in a smooth surface.

where $D_{S}$ is the occluded region on the surface; $X_{v}, Y_{v}, Z_{v}$ are the $X, Y, Z$ coordinates of the original surface mesh with occlusion. We can solve this system of partial differential equations iteratively on the parameter domain:

$$
\begin{cases}\frac{\partial X^{t} \circ \phi}{\partial t}=\frac{1}{\lambda} \operatorname{div}(g(\sqrt{\lambda}|\nabla X \circ \phi|) \nabla X \circ \phi) & \text { on } \phi^{-1}\left(D_{S}\right) ; \\ \frac{\partial Y^{t} \circ \phi}{\partial t}=\frac{1}{\lambda} \operatorname{div}(g(\sqrt{\lambda}|\nabla Y \circ \phi|) \nabla Y \circ \phi) & \text { on } \phi^{-1}\left(D_{S}\right) ; \\ \frac{\partial Z^{t} \circ \phi}{\partial t}=\frac{1}{\lambda} \operatorname{div}(g(\sqrt{\lambda}|\nabla Z \circ \phi|) \nabla Z \circ \phi) & \text { on } \phi^{-1}\left(D_{S}\right) ; \\ X^{0} \circ \phi=X_{v} \circ \phi & \text { on } \phi^{-1}\left(D_{S}^{c}\right) ; \\ Y^{0} \circ \phi=Y_{v} \circ \phi & \text { on } \phi^{-1}\left(D_{S}^{c}\right) ; \\ Z^{0} \circ \phi=Z_{v} \circ \phi & \text { on } \phi^{-1}\left(D_{S}^{c}\right) ;\end{cases}
$$

To illustrate the idea, we test our algorithm to fill in the holes on a human face. Figure 13(A) shows a human face with several holes on it. We applied the surface holes inpainting model to inpaint the occlusion region on the surface. Figure 13(B) shows the inpainted result. As shown in the figure, the occlusion can be filled reasonably well, which results in a smooth surface.

7. Conclusion. In conclusion, we describe a method in this paper to solve variational problems on general surfaces with arbitrary topologies using the global conformal parameterization. With the conformal parameterization of the surface, the problems can be greatly simplified and are transformed into $2 \mathrm{D}$ problems on the parameter domain. The conformal parameterization has a simple metric $\left(g_{i j}\right)=\lambda \mathbf{I} \mathbf{d}$. 
Under the conformal parameterization, the surface differential operators can be computed easily on the $2 \mathrm{D}$ parameter domain with simple formulae. The formulae are very similar to the formulae for the $2 \mathrm{D}$ Euclidean differential operators, except for the scalar multiplication of the conformal factor $\lambda$. Therefore, using the conformal parameterization to transform the variational problems on general surfaces to the $2 \mathrm{D}$ problems on the parameter domain has much easier equations than using other arbitrary parameterizations. The problem can then be solved easily by other well-known numerical schemes.

We have tested our algorithm on solving different image processing and surface processing problems on different surfaces, which require solving different variational problems. The experimental results show that our proposed algorithm can effectively solve the variational problems on the surface. Numerical analysis on the proposed algorithm has also been done to determine how the accuracy of the algorithm is affected by the accuracy in the approximation of the conformal parameterization. In the future, we will look for more applications of solving variational problems and partial differential equations on the surface. In particular, we will look for more applications in medical imaging research area, such as feature detection in human brain mapping research, to study different diseases. Also, based on the numerical analysis we have already done, we will try to develop other better numerical schemes, such as the adpative method, to improve the computational speed and accuracy.

Acknowledgement. We warmly thank Prof. Paul M. Thompson for his valuable advice and friendly help. We are grateful to Dr. Yalin Wang for the fruitful discussion. The brain data used in this work were provided by Center for Computational Biology (CCB), Laboratory of Neuroimaging, UCLA.

\section{REFERENCES}

[1] T.F. Chan And J. Shen, Non-texture inpainting by curvature-driven diffusions (CDD), Journal of Visual Communication and Image Representation, 12:4 (2001), pp. 436-449.

[2] L.A. VESE AND T.F. ChAn, Multiphase level set framework for image segmentation using the Mumford and Shah model, International Journal of Computer Vision, 50:3 (2002), pp. 271293.

[3] L. Rudin, S. Osher, And E. Fatemi, Nonlinear total variation based noise removal algorithms, Physica D: Nonlinear Phenomena, 60:1-4 (1992).

[4] P. Blomgren and T.F. Chan, Color TV: Total variation methods for restoration of vector valued images, IEEE Transaction on Image Processing, 7:3 (1998), pp. 304-309.

[5] T.F. Chan, B.Y. Sandberg, And L.A. Vese, Active contours without edges for vector-valued images, Journal of Visual Communication and Image Representation, 11:2 (2000), pp. 130141.

[6] I.M. Gelfand and S.V. Fomin, Calculus of Variations, Dover, 2000.

[7] X. Gu, Y. Wang, T.F. Chan, P. Thompson, and S.-T. Yau, Genus zero surface conformal mapping and its application to brain surface mapping, IEEE Transaction on Medical Imaging, 23:8 (2004), pp. 949-958.

[8] L. LUi, Y. WANG, T.F. ChAN, AND P. ThOMPSOn, Brain anatomical feature detection by solving partial differential equations on general manifolds, Discrete and Continuous Dynamical Systems B, 7:3 (2007), pp. 605-618.

[9] - Landmark constrained genus zero surface conformal mapping and its application to brain mapping research, Applied Numerical Mathematics, 57 (2007), pp. 847-858.

[10] U. Clarenz, M. Rumpf, and A. Telea, Surface processing methods for point sets using finite elements, Journal of Computers and Graphics, 28:6 (2004), pp. 851-868.

[11] P. Thompson, R. Woods, M.S. Mega, And A. Toga, Mathematical/computational challenges in creating deformable and probablistic atlases of the human brain, IEEE Transactions on Visualization and Computer Graphics (TVGC), 9:2 (2000), pp. 81-92.

[12] P. Thompson, J. Giedd, R. Woods, D. MacDonald, A. Evans, and A. Toga, Growth pat- 
terns in the developing human brain detected using continuum-mechancial tensor mapping, Nature, 404:6774 (2000), pp. 190-193.

[13] L. Lui, Y. WANG, AND T.F. Chan, Solving PDEs on manifold using global conformal parameterization, IEEE Transaction on Medical ImagingVariational, Geometric, and Level Set Methods in Computer Vision: Third International Workshop, VLSM 2005, pp. 307-319, October 2005.

[14] J. Verdera, V. Caselles, M. Bertalmio, and G. Sapiro, Inpainting surface holes, Proceeding of International Conference on Image Processing, 2003 (ICIP 2003), vol. 2, pp. 903-906, September 2003.

[15] J. StAM, Flows on surfaces of arbitrary topology, Proceedings of ACM SIGGRAPH 2003, 22:3 (2003), pp. 724-731.

[16] L. Lopez-Perez, R. Deriche, and N. Sochen, The Beltrami flow over triangulated manifolds, Computer Vision and Mathematical Methods in Medical and Biomedical Image Analysis, 3117 (2004), pp. 135-144.

[17] G. TuRK, Generating textures on arbitrary surfaces using reaction-diffusion, Computer Graphic (SIGGRAPH '91), 25:4 (1991), pp. 289-298.

[18] A. Witkin and M. Kass, Reaction-diffusion textures, Proceedings of the 18th annual conference on computer graphics and interactive techniques, pp. 299-308 (1991).

[19] U. Diewald, T. Preusser, And M. Rumpf, Anisotropic diffusion in vector field visualization on Euclidean domains and surfaces, IEEE Transaction on Visualization and Computer Graphics, 6 (2000), pp. 139-149.

[20] J. Dorsey and P. Hanrahan, Digital materials and virtual weathering, Scientific American, 282:2 (2000), pp. 46-53.

[21] E. Praun, A. Finkelstein, And H. Hoppe, Lapped textures, Proceedings of SiGGRAPH 2000, 2000.

[22] G. Winkenbach and D. Salesin, Rendering parametric surfaces in pen and ink, Computer Graphic (SIGGRAPH 96), pp. 469-476 (1996).

[23] O. Faugeras, F. Clement, R. Deriche, R. Keriven, T. Papadopoulo, J. Roberts, T. Vieville, F. Devernay, J. Gomes, G. Hermosillo, P. Kornprobst, and D. LinGRAND, The inverse EEG and MEG problems: The adjoint state approach I: The continuous case, INRIA Research Report, 3673 (1999).

[24] J. Stam, Stable fluids, Proceedings of the 26th annual conference on computer graphics and interactive techniques, 2002.

[25] J.M. LeE, Riemannian Manifolds, Springer, 1997.

[26] J.M.M. Desbrun and A.N. Hirani, Discrete exterior calculus for variational problems in computer vision and graphics, 42nd IEEE Conference on Decision and Control. Proceedings., 5 (2003), pp. 4902-4907.

[27] M. BuRger, Finite element approximation of elliptic partial differential equations on implicit surfaces, Advances in multiresolution for geometric modelling, 4:56 (2004).

[28] S.O. Marcelo Bertalmio, L.-T. Cheng and G. Sapiro, Variational problems and partial differential equations on implicit surfaces, Journal of Computational Physics, 174:2 (2001), pp. 759-780.

[29] S. RuUth and B. Merriman, A simple embedding method for solving partial differential equations on surfaces, UCLA CAM Report, 6:54 (2006).

[30] M.S. FloAter AND H. KAI, Surface parameterization: a tutorial and survey, Advances in multiresolution for geometric modelling, pp. 157-186 (2005).

[31] A. Ratz And A. Voigt, PDE's on surfaces: a diffuse interface approach, Communications in Mathematical Sciences, 4:3 (2006), pp. 575-590.

[32] Y. Wang, L. Lui, X. Gu, K. Hayashi, T.F. Chan, A. Toga, P. Thompson, and S.-T. YAU, Brain surface conformal parameterization using Riemann surface structure, IEEE Transactions on Medical Imaging, 6 (2007), pp. 853-865.

[33] U. Pinkall and K. Polthier, Computing discrete minimal surfaces and their conjugates, Experimental Mathematics, 2:1 (1993), pp. 15-36.

[34] M. Eck, T. DeRose, T. Duchamp, H. Hoppe, And M. Lounsbery, Multiresolution analysis of arbitrary meshes, Proceedings of the 22nd annual conference on Computer graphics and interactive techniques, pp. 173-182 (1995).

[35] S. Haker, S. Angenent, A. Tannenbaum, R. Kikinis, and M.H.G. Sapiro, Conformal surface parameterization for texture mapping, IEEE Transactions on Visualization and Computer Graphics (TVGC), 6:2 (2000), pp. 181-189.

[36] S. Angenent, S. Haker, A. Tannenbaum, and R. Kikinis, On the Laplace-Beltrami operator and brain surface flattening, IEEE Trans. Medical Imaging, 18 (1999), pp. 700-711.

[37] X. Gu And S.-T. YaU, Computing conformal structures of surfaces, Communications in In- 
formation and Systems, 2:2 (2002), pp. 121-146.

[38] — Global conformal surface parameterization, ACM Symposium on Geometry Processing 2003, 2003.

[39] S. WAng, Y. WAng, M. Jin, X. Gu, And D. Samaras, Conformal geometry and its applications on 3D shape matching, recognition and stitching, IEEE Transactions on Pattern Analysis and Machine Intelligence, 29:7 (2007), pp. 1209-1220.

[40] L. Lopez-Perez, R. Deriche, and N. Sochen, The Beltrami flow over triangulated manifolds, Computer Vision and Mathematical Methods in Medical and Biomedical Image Analysis, 3117 (2004), pp. 135-144.

[41] T.F. Chan And J. Shen, Variational restoration of nonflat image features: Models and algorithms, SIAM Journal on Applied Mathematics, 61:4 (2000), pp. 1338-1361.

[42] B. TANG, G. SAPIRO, AND V. CASElles, Diffusion of general data on non-flat manifolds via harmonic maps theory: The direction diffusion case, International Journal of Computer Vision, 36:2 (2000), pp. 149-161.

[43] A. SpIRA AND R. Kimmel, Enhancing images painted on manifolds, Scale Space and PDE Methods in Computer Vision, 3459 (2005), pp. 492-502.

[44] T.P. Breckon AND R.B. Fisher, Direct geometric texture synthesis and transfer on 3D meshes, 3rd European Conference on Visual Media Production (CVMP), pp. 186-186 (2006).

[45] L.M. Lui, Y. Wang, T.F. Chan, And P. Thompson, Automatic landmark tracking and its application to the optimization of brain conformal mapping, IEEE Computer Society Conference on Computer Vision and Pattern Recognition (CVPR), New York, 2 (2006), pp. 17841792.

[46] T.P. Breckon and R.B. Fisher, Non-parametric 3D surface completion, Proceedings of the Fifth International Conference on 3-D Digital Imaging and Modeling, pp. 573-580 (2005). 